\title{
Lattice chiral effective field theory with three-body interactions at next-to-next-to-leading order
}

\author{
Evgeny Epelbaum ${ }^{a, b}$, Hermann Krebs ${ }^{b, a}$, Dean Lee ${ }^{c, b}$, Ulf-G. Meißner ${ }^{b, a, d}$ \\ ${ }^{a}$ Institut für Kernphysik (IKP-3) and Jülich Center for Hadron Physics, \\ Forschungszentrum Jülich, D-52425 Jülich, Germany \\ ${ }^{b}$ Helmholtz-Institut für Strahlen- und Kernphysik (Theorie) \\ and Bethe Center for Theoretical Physics, \\ Universität Bonn, D-53115 Bonn, Germany \\ ${ }^{c}$ Department of Physics, North Carolina State University, Raleigh, NC 27695, USA \\ ${ }^{d}$ Institute for Advanced Simulations (IAS), \\ Forschungszentrum Jülich, D-52425 Jülich, Germany
}

\begin{abstract}
We consider low-energy nucleons at next-to-next-to-leading order in lattice chiral effective field theory. Three-body interactions first appear at this order, and we discuss several methods for determining three-body interaction coefficients on the lattice. We compute the energy of the triton and low-energy neutron-deuteron scattering phase shifts in the spindoublet and spin-quartet channels using Lüscher's finite volume method. In the four-nucleon system we calculate the energy of the $\alpha$ particle using auxiliary fields and projection Monte Carlo.
\end{abstract}




\section{INTRODUCTION}

We study low-energy nucleons on the lattice at next-to-next-to-leading order in chiral effective field theory. In Weinberg's scheme [1, 2] counting orders in effective field theory is equivalent to dimensional analysis for irreducible diagrams. The expansion parameter is $Q / \Lambda$, where $Q$ is the low momentum scale associated with external nucleon momenta or the pion mass, and $\Lambda$ is the high momentum scale at which the effective theory breaks down. Terms at next-to-next-to-leading order are of size $Q^{3} / \Lambda^{3}$, and three-nucleon interactions first contribute at this order. In this work we consider three-nucleon forces on the lattice for systems with three and four nucleons. Our analysis continues a series of recent papers on lattice chiral effective field theory for few- and many-nucleon systems. Previous studies have considered dilute neutron matter and light nuclei using interactions at leading order [3, 4] and next-to-leading order [5, 6, 7].

Our discussion is organized into three parts. The first part begins with an overview of the effective potential for nucleons up to next-to-next-to-leading order in chiral effective field theory. Reviews of chiral effective field theory can be found in Ref. [8, 9, 10, 11]. We discuss some simplifications that can be made at low cutoff momentum and present lattice operators for each interaction. Nucleon-nucleon phase shifts and the $S-D$ mixing angle are determined using a spherical wall method [12] and used to set unknown operator coefficients. In the second part we calculate the low-energy spectrum for three nucleons. We compute the triton energy and determine neutron-deuteron phase shifts using Lüscher's finite volume method. These are used to constrain the two unknown three-body operator coefficients. In the third and final part we rewrite the lattice action in terms of auxiliary fields and use projection Monte Carlo to calculate the energy of the $\alpha$ particle. This leads to a discussion of alternative methods for fixing three-body operator coefficients. We summarize our results and discuss possible extensions in future work.

\section{CHIRAL EFFECTIVE FIELD THEORY}

\section{A. Effective potential for two nucleons}

In the following $\vec{q}$ denotes the $t$-channel momentum transfer for nucleon-nucleon scattering while $\vec{k}$ is the $u$-channel exchanged momentum transfer. We assume exact isospin symmetry 
and neglect electromagnetic interactions. At leading order (LO) in the Weinberg scheme the two-nucleon effective potential consists of two independent contact terms and instantaneous one-pion exchange (OPEP),

$$
V_{\mathrm{LO}}=V^{(0)}+V^{\mathrm{OPEP}} .
$$

The scattering between nucleons consists of contributions from direct and exchange diagrams. Nevertheless for bookkeeping purposes we label the interactions according to the tree-level scattering amplitude for distinguishable nucleons. For two-nucleon interactions we label one nucleon as type $A$ and the other nucleon as type $B$. In this notation the amplitude for $V^{(0)}$ is

$$
\mathcal{A}\left[V^{(0)}\right]=C_{S}+C_{T}\left(\vec{\sigma}_{A} \cdot \vec{\sigma}_{B}\right),
$$

and the amplitude for $V^{\mathrm{OPEP}}$ is

$$
\mathcal{A}\left[V^{\mathrm{OPEP}}\right]=-\left(\frac{g_{A}}{2 f_{\pi}}\right)^{2} \boldsymbol{\tau}_{A} \cdot \boldsymbol{\tau}_{B} \frac{\left(\vec{\sigma}_{A} \cdot \vec{q}\right)\left(\vec{\sigma}_{B} \cdot \vec{q}\right)}{q^{2}+m_{\pi}^{2}} .
$$

The vector arrow in $\vec{\sigma}$ signifies the three-vector index for spin. The boldface for $\boldsymbol{\tau}$ signifies the three-vector index for isospin. We take for our physical constants $m=938.92 \mathrm{MeV}$ as the nucleon mass, $m_{\pi}=138.08 \mathrm{MeV}$ as the pion mass, $f_{\pi}=93 \mathrm{MeV}$ as the pion decay constant, and $g_{A}=1.26$ as the nucleon axial charge.

At next-to-leading order (NLO) the two-nucleon effective potential contains seven independent contact terms carrying two powers of momentum, corrections to the two LO contact terms, and the leading contribution from the instantaneous two-pion exchange potential (TPEP) [13, 14, 15, 16, 17],

$$
V_{\mathrm{NLO}}=V_{\mathrm{LO}}+\Delta V^{(0)}+V^{(2)}+V_{\mathrm{NLO}}^{\mathrm{TPEP}} .
$$

The tree-level amplitudes for the contact interactions are

$$
\mathcal{A}\left[\Delta V^{(0)}\right]=\Delta C_{S}+\Delta C_{T}\left(\vec{\sigma}_{A} \cdot \vec{\sigma}_{B}\right)
$$

and

$$
\begin{aligned}
\mathcal{A}\left[V^{(2)}\right] & =C_{1} q^{2}+C_{2} k^{2}+\left(C_{3} q^{2}+C_{4} k^{2}\right)\left(\vec{\sigma}_{A} \cdot \vec{\sigma}_{B}\right)+i C_{5} \frac{1}{2}\left(\vec{\sigma}_{A}+\vec{\sigma}_{B}\right) \cdot(\vec{q} \times \vec{k}) \\
& +C_{6}\left(\vec{\sigma}_{A} \cdot \vec{q}\right)\left(\vec{\sigma}_{B} \cdot \vec{q}\right)+C_{7}\left(\vec{\sigma}_{A} \cdot \vec{k}\right)\left(\vec{\sigma}_{B} \cdot \vec{k}\right) .
\end{aligned}
$$


The amplitude for the NLO two-pion exchange potential is [18, 19]

$$
\begin{aligned}
\mathcal{A}\left[V_{\mathrm{NLO}}^{\mathrm{TPEP}}\right] & =-\frac{\boldsymbol{\tau}_{A} \cdot \boldsymbol{\tau}_{B}}{384 \pi^{2} f_{\pi}^{4}} L(q)\left[4 m_{\pi}^{2}\left(5 g_{A}^{4}-4 g_{A}^{2}-1\right)+q^{2}\left(23 g_{A}^{4}-10 g_{A}^{2}-1\right)+\frac{48 g_{A}^{4} m_{\pi}^{4}}{4 m_{\pi}^{2}+q^{2}}\right] \\
& -\frac{3 g_{A}^{4}}{64 \pi^{2} f_{\pi}^{4}} L(q)\left[\left(\vec{q} \cdot \vec{\sigma}_{A}\right)\left(\vec{q} \cdot \vec{\sigma}_{B}\right)-q^{2}\left(\vec{\sigma}_{A} \cdot \vec{\sigma}_{B}\right)\right]
\end{aligned}
$$

where

$$
L(q)=\frac{1}{2 q} \sqrt{4 m_{\pi}^{2}+q^{2}} \ln \frac{\sqrt{4 m_{\pi}^{2}+q^{2}}+q}{\sqrt{4 m_{\pi}^{2}+q^{2}}-q} .
$$

At next-to-next-to-leading order (NNLO) there are no additional two-nucleon contact interactions, but the two-pion exchange potential contains a subleading contribution,

$$
\begin{aligned}
\mathcal{A}\left[V_{\mathrm{NNLO}}^{\mathrm{TPEP}}\right] & =-\frac{3 g_{A}^{2}}{16 \pi f_{\pi}^{4}} A(q)\left(2 m_{\pi}^{2}+q^{2}\right)\left[2 m_{\pi}^{2}\left(2 c_{1}-c_{3}\right)-c_{3} q^{2}\right] \\
& -\frac{g_{A}^{2} c_{4}\left(\boldsymbol{\tau}_{A} \cdot \boldsymbol{\tau}_{B}\right)}{32 \pi f_{\pi}^{4}} A(q)\left(4 m_{\pi}^{2}+q^{2}\right)\left[\left(\vec{q} \cdot \vec{\sigma}_{A}\right)\left(\vec{q} \cdot \vec{\sigma}_{B}\right)-q^{2}\left(\vec{\sigma}_{A} \cdot \vec{\sigma}_{B}\right)\right],
\end{aligned}
$$

where

$$
A(q)=\frac{1}{2 q} \arctan \frac{q}{2 m_{\pi}} .
$$

The low-energy constants $c_{1}, c_{3}, c_{4}$ parameterize the coupling of the nucleon to two pions. These constants have been determined from fits to low-energy pion-nucleon scattering data [20], and in the following we use the values $c_{1}=-0.81 \mathrm{GeV}^{-1}, c_{3}=-4.7 \mathrm{GeV}^{-1}, c_{4}=$ $3.4 \mathrm{GeV}^{-1}[21]$.

\section{B. Three-nucleon interactions}

A number of different phenomenological three-nucleon potentials have been introduced in the literature [22, 23, 24, 25, 26, 27, 28, 29, 30]. Effective field theory provides a systematic method for estimating the relative importance of three-body interaction terms. Few-nucleon forces in chiral effective field theory beyond two nucleons were first introduced in Ref. [2]. In Ref. [31] it was shown that three-nucleon interactions at NLO cancel and three-body effects first appear at NNLO. The NNLO three-nucleon effective potential includes a pure contact potential, $V_{\text {contact }}^{(3 N)}$, one-pion exchange potential, $V_{\mathrm{OPE}}^{(3 N)}$, and a two-pion exchange potential, $V_{\mathrm{TPE}}^{(3 N)}$

$$
V_{\mathrm{NNLO}}^{(3 N)}=V_{\text {contact }}^{(3 N)}+V_{\mathrm{OPE}}^{(3 N)}+V_{\mathrm{TPE}}^{(3 N)}
$$

The corresponding diagrams are shown in Fig. 1, 


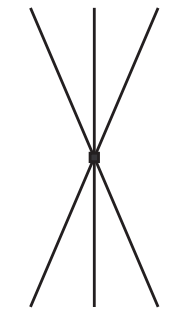

(a)

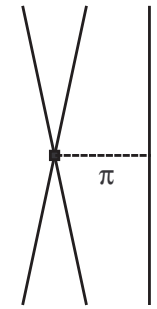

(b)

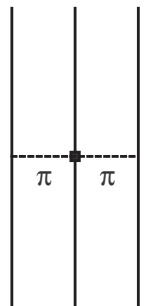

(c)

FIG. 1: Three-nucleon forces at NNLO. Diagrams (a), (b), and (c) show the contact potential, $V_{\text {contact }}^{(3 N)}$, one-pion exchange potential $V_{\mathrm{OPE}}^{(3 N)}$, and two-pion exchange potential $V_{\mathrm{TPE}}^{(3 N)}$.

Similar to our bookkeeping notation for two-nucleon interactions, we write the tree-level amplitude for three-nucleon interactions where the first nucleon is type $A$, the second nucleon type $B$, and the third type $C$. We sum over all permutations $P(A, B, C)$ of the labels, and $\vec{q}_{A}, \vec{q}_{B}, \vec{q}_{C}$ are defined as the differences between final and initial momenta for the respective nucleons. The amplitudes for $V_{\text {contact }}^{(3 N)}$ and $V_{\mathrm{OPE}}^{(3 N)}$ are [32, 33 ]

$$
\begin{gathered}
\mathcal{A}\left[V_{\text {contact }}^{(3 N)}\right]=\frac{1}{2} E \sum_{P(A, B, C)}\left(\boldsymbol{\tau}_{A} \cdot \boldsymbol{\tau}_{B}\right), \\
\mathcal{A}\left[V_{\mathrm{OPE}}^{(3 N)}\right]=-\frac{g_{A}}{8 f_{\pi}^{2}} D \sum_{P(A, B, C)} \frac{\vec{q}_{A} \cdot \vec{\sigma}_{A}}{q_{A}^{2}+m_{\pi}^{2}}\left(\vec{q}_{A} \cdot \vec{\sigma}_{B}\right)\left(\boldsymbol{\tau}_{A} \cdot \boldsymbol{\tau}_{B}\right) .
\end{gathered}
$$

The coefficients $E$ and $D$ are both cutoff dependent. The coefficient $E$ determines the short distance interactions between three nucleons, while $D$ determines the pion coupling to two nucleons. Following the notation introduced in Ref. [33], we define dimensionless parameters $c_{E}$ and $c_{D}$ such that

$$
E=\frac{c_{E}}{f_{\pi}^{4} \Lambda_{\chi}}, \quad D=\frac{c_{D}}{f_{\pi}^{2} \Lambda_{\chi}},
$$

where $\Lambda_{\chi} \simeq m_{\rho}$. We take $\Lambda_{\chi}=700 \mathrm{MeV}$.

For convenience we separately label three parts of the two-pion exchange potential,

$$
V_{\mathrm{TPE}}^{(3 N)}=V_{\mathrm{TPE} 1}^{(3 N)}+V_{\mathrm{TPE} 2}^{(3 N)}+V_{\mathrm{TPE} 3}^{(3 N)} .
$$

The corresponding amplitudes are

$$
\mathcal{A}\left[V_{\mathrm{TPE} 1}^{(3 N)}\right]=\frac{c_{3}}{f_{\pi}^{2}}\left(\frac{g_{A}}{2 f_{\pi}}\right)^{2} \sum_{P(A, B, C)} \frac{\left(\vec{q}_{A} \cdot \vec{\sigma}_{A}\right)\left(\vec{q}_{B} \cdot \vec{\sigma}_{B}\right)}{\left(q_{A}^{2}+m_{\pi}^{2}\right)\left(q_{B}^{2}+m_{\pi}^{2}\right)}\left(\vec{q}_{A} \cdot \vec{q}_{B}\right)\left(\boldsymbol{\tau}_{A} \cdot \boldsymbol{\tau}_{B}\right),
$$




$$
\begin{aligned}
& \mathcal{A}\left[V_{\mathrm{TPE} 2}^{(3 N)}\right]=-\frac{2 c_{1} m_{\pi}^{2}}{f_{\pi}^{2}}\left(\frac{g_{A}}{2 f_{\pi}}\right)^{2} \sum_{P(A, B, C)} \frac{\left(\vec{q}_{A} \cdot \vec{\sigma}_{A}\right)\left(\vec{q}_{B} \cdot \vec{\sigma}_{B}\right)}{\left(q_{A}^{2}+m_{\pi}^{2}\right)\left(q_{B}^{2}+m_{\pi}^{2}\right)}\left(\boldsymbol{\tau}_{A} \cdot \boldsymbol{\tau}_{B}\right), \\
& \mathcal{A}\left[V_{\mathrm{TPE} 3}^{(3 N)}\right]= \frac{c_{4}}{2 f_{\pi}^{2}}\left(\frac{g_{A}}{2 f_{\pi}}\right)^{2} \\
& \times \sum_{P(A, B, C)} \frac{\left(\vec{q}_{A} \cdot \vec{\sigma}_{A}\right)\left(\vec{q}_{B} \cdot \vec{\sigma}_{B}\right)}{\left(q_{A}^{2}+m_{\pi}^{2}\right)\left(q_{B}^{2}+m_{\pi}^{2}\right)}\left[\left(\vec{q}_{A} \times \vec{q}_{B}\right) \cdot \vec{\sigma}_{C}\right]\left[\left(\boldsymbol{\tau}_{A} \times \boldsymbol{\tau}_{B}\right) \cdot \boldsymbol{\tau}_{C}\right] .
\end{aligned}
$$

\section{Simplified form at low cutoff momentum}

A number of alternatives to Weinberg's power counting have recently been discussed in the literature [34, 35, 36, 37]. However at low cutoff momentum the advantages of these alternative schemes are numerically small [38]. In this study we use spatial lattice spacing $a=(100 \mathrm{MeV})^{-1}$, corresponding with cutoff momentum $\Lambda=314 \mathrm{MeV} \approx 2.3 m_{\pi}$. Our choice of low cutoff scale avoids numerical problems in Monte Carlo simulations due to spurious deeply-bound states and large sign/phase oscillations.

In the following lattice calculations we use Weinberg's power counting with some additional simplifications made possible by the low cutoff momentum. For nearly all $|q|<\Lambda$ we can expand the NLO two-pion exchange potential in powers of $q^{2} /\left(4 m_{\pi}^{2}\right)$,

$$
\begin{aligned}
\mathcal{A}\left[V_{\mathrm{NLO}}^{\mathrm{TPEP}}\right] & =-\frac{\boldsymbol{\tau}_{A} \cdot \boldsymbol{\tau}_{B}}{384 \pi^{2} f_{\pi}^{4}}\left[4 m_{\pi}^{2}\left(8 g_{A}^{4}-4 g_{A}^{2}-1\right)+\frac{2}{3} q^{2}\left(34 g_{A}^{4}-17 g_{A}^{2}-2\right)+m_{\pi}^{2} O\left(\left(\frac{q^{2}}{4 m_{\pi}^{2}}\right)^{2}\right)\right] \\
& -\frac{3 g_{A}^{4}}{64 \pi^{2} f_{\pi}^{4}}\left[\left(\vec{q} \cdot \vec{\sigma}_{A}\right)\left(\vec{q} \cdot \vec{\sigma}_{B}\right)-q^{2}\left(\vec{\sigma}_{A} \cdot \vec{\sigma}_{B}\right)\right]\left[1+O\left(\frac{q^{2}}{4 m_{\pi}^{2}}\right)\right]
\end{aligned}
$$

This expansion fails to converge only for values of $q$ near the cutoff scale $\Lambda \approx 2.3 m_{\pi}$, where the effective theory is already problematic due to large cutoff effects. From a practical viewpoint there is no advantage in retaining the full non-local structure of $V_{\text {NLO }}^{\text {TPEP }}$ at this lattice spacing. Instead we simply use

$$
\begin{gathered}
V_{\mathrm{LO}}=V^{(0)}+V^{\mathrm{OPEP}}, \\
V_{\mathrm{NLO}}=V_{\mathrm{LO}}+\Delta V^{(0)}+V^{(2)},
\end{gathered}
$$

where the terms in Eq. (19) with up to two powers of $q$ are absorbed as a redefinition of the coefficients $\Delta V^{(0)}$ and $V^{(2)}$. 
Similarly we can expand the NNLO two-pion exchange potential,

$$
\begin{aligned}
\mathcal{A}\left[V_{\mathrm{NNLO}}^{\mathrm{TPEP}}\right] & =-\frac{3 g_{A}^{2} m_{\pi}}{16 \pi f_{\pi}^{4}}\left[m_{\pi}^{2}\left(2 c_{1}-c_{3}\right)+q^{2}\left(\frac{5}{6} c_{1}-\frac{11}{12} c_{3}\right)+m_{\pi}^{2} O\left(\left(\frac{q^{2}}{4 m_{\pi}^{2}}\right)^{2}\right)\right] \\
& -\frac{g_{A}^{2} c_{4}\left(\boldsymbol{\tau}_{A} \cdot \boldsymbol{\tau}_{B}\right) m_{\pi}}{32 \pi f_{\pi}^{4}}\left[\left(\vec{q} \cdot \vec{\sigma}_{A}\right)\left(\vec{q} \cdot \vec{\sigma}_{B}\right)-q^{2}\left(\vec{\sigma}_{A} \cdot \vec{\sigma}_{B}\right)\right]\left[1+O\left(\frac{q^{2}}{4 m_{\pi}^{2}}\right)\right] .
\end{aligned}
$$

The terms with two powers of $q$ were already included at NLO, and so there are no additional terms in the two-nucleon potential at NNLO. In our low cutoff scheme the only new contributions at NNLO are due to three-nucleon interactions,

$$
V_{\mathrm{NNLO}}=V_{\mathrm{NLO}}+V_{\mathrm{NNLO}}^{(3 N)}
$$

\section{LATTICE INTERACTIONS AT LO AND NLO}

\section{A. Transfer matrix at LO}

In our Euclidean-time lattice formalism the transfer matrix operator is the normal-ordered exponential of the lattice Hamiltonian, : $\exp (-H \Delta t):$, where $\Delta t$ equals one temporal lattice spacing, $a_{t}$. At leading order we use the $\mathrm{LO}_{2}$ transfer matrix with Gaussian-smeared

interactions [4, 5, 6]. Since we consider only one action, we drop the "2" subscript on $\mathrm{LO}_{2}$. The transfer matrix operator is

$$
\begin{aligned}
M_{\mathrm{LO}} & =: \exp \left\{-H_{\text {free }} \alpha_{t}-\frac{\alpha_{t}}{2 L^{3}} \sum_{\vec{q}} f(\vec{q})\left[C \rho^{a^{\dagger}, a}(\vec{q}) \rho^{a^{\dagger}, a}(-\vec{q})+C_{I^{2}} \sum_{I} \rho_{I}^{a^{\dagger}, a}(\vec{q}) \rho_{I}^{a^{\dagger}, a}(-\vec{q})\right]\right. \\
& \left.+\frac{g_{A}^{2} \alpha_{t}^{2}}{8 f_{\pi}^{2} q_{\pi}} \sum_{S_{1}, S_{2}, I} \sum_{\vec{n}_{1}, \vec{n}_{2}} G_{S_{1} S_{2}}\left(\vec{n}_{1}-\vec{n}_{2}\right) \rho_{S_{1}, I}^{a^{\dagger}, a}\left(\vec{n}_{1}\right) \rho_{S_{2}, I}^{a^{\dagger}, a}\left(\vec{n}_{2}\right)\right\}:
\end{aligned}
$$

The momentum-dependent coefficient function $f(\vec{q})$ is given by

$$
f(\vec{q})=f_{0}^{-1} \exp \left[-b \sum_{l}\left(1-\cos q_{l}\right)\right],
$$

where

$$
f_{0}=\frac{1}{L^{3}} \sum_{\vec{q}} \exp \left[-b \sum_{l}\left(1-\cos q_{l}\right)\right] .
$$

Our lattice notation is defined in the Appendix. The densities $\rho^{a^{\dagger}, a}$ and $\rho_{I}^{a^{\dagger}, a}$ and spindependent one-pion exchange potential $G_{S_{1} S_{2}}$ are also defined in the Appendix. We use 


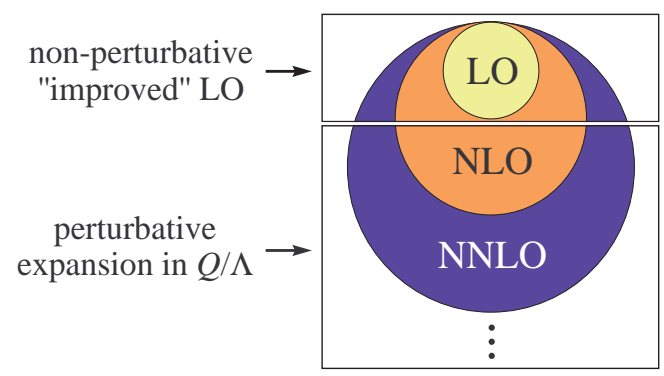

FIG. 2: The "improved" LO action is iterated non-perturbatively while the remaining higher-order interactions are treated using perturbation theory.

the value $b=0.6$, which gives approximately the correct effective range for the two $S$-wave channels when $C$ and $C_{I^{2}}$ are tuned to the physical scattering lengths. $C$ is the coefficient of the Wigner SU(4)-invariant contact interaction [39], and $C_{I^{2}}$ is the coefficient of the isospindependent contact interaction. In terms of coefficients for the isospin-singlet and triplet channels,

$$
\begin{gathered}
C=\left(3 C^{I=1}+C^{I=0}\right) / 4, \\
C_{I^{2}}=\left(C^{I=1}-C^{I=0}\right) / 4 .
\end{gathered}
$$

This "improved" leading-order action is treated non-perturbatively while higher-order interactions are included as a perturbative expansion in powers of $Q / \Lambda$. This is sketched in Fig. 2.

In pionless effective field theory the three-nucleon contact interaction is included at leading order [40, 41, 42]. This is needed to stabilize the three-nucleon system in the limit of zero-range interactions [43]. In this study we use chiral effective field theory where the interactions have nonzero range. However given our coarse lattice spacing, we may find that the three-body contact interaction is numerically large and requires non-perturbative treatment. Non-perturbative three-body contact interactions on the lattice have been discussed in the literature [44, 45, 46]. However for the lattice calculations presented here we choose a different approach to address the same problem.

We use the fact that the three-nucleon interaction depends on both the spatial lattice spacing, $a$, and the temporal lattice spacing, $a_{t}$. The temporal lattice spacing regulates the transfer matrix element when the interaction potential energy exceeds $a_{t}^{-1}$. As a result 
it can affect the magnitude and sign of the three-body contact interaction [47]. With the spatial lattice spacing held fixed, we dial the temporal lattice spacing to a value where the three-nucleon interaction is numerically small. This involves a calculation of the spectrum of the three-nucleon system. In the following calculations we use the lattice spacings $a=(100 \mathrm{MeV})^{-1}$ and $a_{t}=(150 \mathrm{MeV})^{-1}$ and show that the strength of the three-nucleon

contact interaction is small enough to be treated using perturbation theory. For these lattice spacings we find leading-order coefficients $C^{I=0}=-5.105 \times 10^{-5} \mathrm{MeV}^{-2}$ and $C^{I=1}=$ $-3.507 \times 10^{-5} \mathrm{MeV}^{-2}$ when tuned to the physical $S$-wave scattering lengths.

\section{B. Transfer matrix at NLO}

At next-to-leading order the lattice transfer matrix is

$$
\begin{aligned}
M_{\mathrm{NLO}}=M_{\mathrm{LO}}-\alpha_{t}:[\Delta V & +\Delta V_{I^{2}}+V_{q^{2}}+V_{I^{2}, q^{2}}+V_{S^{2}, q^{2}} \\
& \left.+V_{S^{2}, I^{2}, q^{2}}+V_{(q \cdot S)^{2}}+V_{I^{2},(q \cdot S)^{2}}+V_{(i q \times S) \cdot k}^{I=1}\right] M_{\mathrm{LO}}: .
\end{aligned}
$$

The corrections to the leading-order contact interactions are

$$
\begin{aligned}
\Delta V & =\frac{1}{2} \Delta C: \sum_{\vec{n}} \rho^{a^{\dagger}, a}(\vec{n}) \rho^{a^{\dagger}, a}(\vec{n}): \\
\Delta V_{I^{2}} & =\frac{1}{2} \Delta C_{I^{2}}: \sum_{\vec{n}, I} \rho_{I}^{a^{\dagger}, a}(\vec{n}) \rho_{I}^{a^{\dagger}, a}(\vec{n}):,
\end{aligned}
$$

and the seven independent contact interactions with two derivatives are

$$
\begin{gathered}
V_{q^{2}}=-\frac{1}{2} C_{q^{2}}: \sum_{\vec{n}, l} \rho^{a^{\dagger}, a}(\vec{n}) \nabla_{l}^{2} \rho^{a^{\dagger}, a}(\vec{n}): \\
V_{I^{2}, q^{2}}=-\frac{1}{2} C_{I^{2}, q^{2}}: \sum_{\vec{n}, I, l} \rho_{I}^{a^{\dagger}, a}(\vec{n}) \nabla_{l}^{2} \rho_{I}^{a^{\dagger}, a}(\vec{n}): \\
V_{S^{2}, q^{2}}=-\frac{1}{2} C_{S^{2}, q^{2}}: \sum_{\vec{n}, S, l} \rho_{S}^{a^{\dagger}, a}(\vec{n}) \nabla_{l}^{2} \rho_{S}^{a^{\dagger}, a}(\vec{n}): \\
V_{S^{2}, I^{2}, q^{2}}=-\frac{1}{2} C_{S^{2}, I^{2}, q^{2}}: \sum_{\vec{n}, S, I, l} \rho_{S, I}^{a^{\dagger}, a}(\vec{n}) \nabla_{l}^{2} \rho_{S, I}^{a^{\dagger}, a}(\vec{n}): \\
V_{(q \cdot S)^{2}}=\frac{1}{2} C_{(q \cdot S)^{2}}: \sum_{\vec{n}} \sum_{S} \Delta_{S} \rho_{S}^{a^{\dagger}, a}(\vec{n}) \sum_{S^{\prime}} \Delta_{S^{\prime}} \rho_{S^{\prime}}^{a^{\dagger}, a}(\vec{n}):
\end{gathered}
$$




$$
\begin{gathered}
V_{I^{2},(q \cdot S)^{2}}=\frac{1}{2} C_{I^{2},(q \cdot S)^{2}}: \sum_{\vec{n}, I} \sum_{S} \Delta_{S} \rho_{S, I}^{a^{\dagger}, a}(\vec{n}) \sum_{S^{\prime}} \Delta_{S^{\prime}} \rho_{S^{\prime}, I}^{a^{\dagger}, a}(\vec{n}): \\
V_{(i q \times S) \cdot k}^{I=1}=-\frac{i}{2} C_{(i q \times S) \cdot k}^{I=1}\left\{\frac{3}{4}: \sum_{\vec{n}, l, S, l^{\prime}} \varepsilon_{l, S, l^{\prime}}\left[\prod_{l}^{a^{\dagger}, a}(\vec{n}) \Delta_{l^{\prime}} \rho_{S}^{a^{\dagger}, a}(\vec{n})+\Pi_{l, S}^{a^{\dagger}, a}(\vec{n}) \Delta_{l^{\prime}} \rho^{a^{\dagger}, a}(\vec{n})\right]:\right. \\
\left.+\frac{1}{4}: \sum_{\vec{n}, l, S, l^{\prime}, I} \varepsilon_{l, S, l^{\prime}}\left[\prod_{l, I}^{a^{\dagger}, a}(\vec{n}) \Delta_{l^{\prime}} \rho_{S, I}^{a^{\dagger}, a}(\vec{n})+\Pi_{l, S, I}^{a^{\dagger}, a}(\vec{n}) \Delta_{l^{\prime}} \rho_{I}^{a^{\dagger}, a}(\vec{n})\right]:\right\} .
\end{gathered}
$$

The densities, current densities, and symbols $\Delta_{l}$ and $\nabla_{l}^{2}$, are defined in the Appendix.

The $V_{(i q \times S) \cdot k}^{I=1}$ term is designed to eliminate lattice artifacts in the spin-triplet even-parity channels. This is done by projecting onto the isospin-triplet state,

$$
V_{(i q \times S) \cdot k}^{I=1}=V_{(i q \times S) \cdot k}+V_{I^{2},(i q \times S) \cdot k},
$$

where

$$
\begin{gathered}
V_{(i q \times S) \cdot k}=-\frac{i}{2} C_{(i q \times S) \cdot k}: \sum_{\vec{n}, l, S, l^{\prime}} \varepsilon_{l, S, l^{\prime}}\left[\Pi_{l}^{a^{\dagger}, a}(\vec{n}) \Delta_{l^{\prime}} \rho_{S}^{a^{\dagger}, a}(\vec{n})+\Pi_{l, S}^{a^{\dagger}, a}(\vec{n}) \Delta_{l^{\prime}} \rho^{a^{\dagger}, a}(\vec{n})\right]: \\
V_{I^{2},(i q \times S) \cdot k}=-\frac{i}{2} C_{I^{2},(i q \times S) \cdot k}: \sum_{\vec{n}, l, S, l^{\prime}, I} \varepsilon_{l, S, l^{\prime}}\left[\prod_{l, I}^{a^{\dagger}, a}(\vec{n}) \Delta_{l^{\prime}} \rho_{S, I}^{a^{\dagger}, a}(\vec{n})+\Pi_{l, S, I}^{a^{\dagger}, a}(\vec{n}) \Delta_{l^{\prime}} \rho_{I}^{a^{\dagger}, a}(\vec{n})\right]:,
\end{gathered}
$$

and

$$
\begin{gathered}
C_{(i q \times S) \cdot k}=\frac{3}{4} C_{(i q \times S) \cdot k}^{I=1}, \\
C_{I^{2},(i q \times S) \cdot k}=\frac{1}{4} C_{(i q \times S) \cdot k}^{I=1} .
\end{gathered}
$$

We measure phase shifts on the lattice by imposing a spherical wall boundary on the relative separation between two nucleons at some chosen radius. From the properties of the spherical standing waves we determine scattering phase shifts and mixing angles [12]. The scattering results are nearly identical with the $\mathrm{LO}_{2}$ data at lattice spacings $a=(100 \mathrm{MeV})^{-1}$ and $a_{t}=(70 \mathrm{MeV})^{-1}$ presented in Ref. [5]. The values for the next-to-leading order coefficients are shown in Table II. These values are similar to the $\mathrm{NLO}_{2}$ coefficients in Table III of Ref. [5], though there are some differences due to the change in temporal lattice spacing. 
TABLE I: Results for the NLO operator coefficients

\begin{tabular}{||c|c||}
\hline \hline Coefficient & Value \\
\hline$\Delta C\left[\mathrm{MeV}^{-2}\right]$ & $4.08 \times 10^{-6}$ \\
\hline$\Delta C_{I^{2}}\left[\mathrm{MeV}^{-2}\right]$ & $5.92 \times 10^{-6}$ \\
\hline$C_{q^{2}}\left[\mathrm{MeV}^{-4}\right]$ & $-1.31 \times 10^{-9}$ \\
\hline$C_{I^{2}, q^{2}}\left[\mathrm{MeV}^{-4}\right]$ & $-3.26 \times 10^{-10}$ \\
\hline$C_{S^{2}, q^{2}}\left[\mathrm{MeV}^{-4}\right]$ & $-1.53 \times 10^{-10}$ \\
\hline$C_{S^{2}, I^{2}, q^{2}}\left[\mathrm{MeV}^{-4}\right]$ & $-2.64 \times 10^{-10}$ \\
\hline$C_{(q \cdot S)^{2}}\left[\mathrm{MeV}^{-4}\right]$ & $-1.92 \times 10^{-10}$ \\
\hline$C_{I^{2},(q \cdot S)^{2}}\left[\mathrm{MeV}^{-4}\right]$ & $9.20 \times 10^{-12}$ \\
\hline$C_{(i q \times S) \cdot k}^{I=1}\left[\mathrm{MeV}^{-4}\right]$ & $1.11 \times 10^{-10}$ \\
\hline \hline
\end{tabular}

\section{THREE-NUCLEON INTERACTIONS AT NNLO}

At next-to-next-to-leading order the transfer matrix is

$$
M_{\mathrm{NNLO}}=M_{\mathrm{NLO}}-\alpha_{t}:\left[V_{\text {contact }}^{(3 N)}+V_{\mathrm{OPE}}^{(3 N)}+V_{\mathrm{TPE} 1}^{(3 N)}+V_{\mathrm{TPE} 2}^{(3 N)}+V_{\mathrm{TPE} 3}^{(3 N)}\right] M_{\mathrm{LO}}: .
$$

From the constraints of isospin symmetry, spin symmetry, and Fermi statistics, there is only one independent three-nucleon contact interaction [33, 42]. In Eq. (12) we wrote this as a $\boldsymbol{\tau}_{A} \cdot \boldsymbol{\tau}_{B}$ interaction over all permutations of the labels $A, B, C$. For our lattice action we choose to write the contact interaction $V_{\text {contact }}^{(3 N)}$ as a product of total nucleon densities,

$$
V_{\text {contact }}^{(3 N)}=\frac{1}{6} D_{\text {contact }}: \sum_{\vec{n}}\left[\rho^{a^{\dagger}, a}(\vec{n})\right]^{3}:
$$

The one-pion exchange potential $V_{\mathrm{OPE}}^{(3 N)}$ can be written as

$$
V_{\mathrm{OPE}}^{(3 N)}=-D_{\mathrm{OPE}} \frac{g_{A} \alpha_{t}}{2 f_{\pi} q_{\pi}} \sum_{\vec{n}, S, I} \sum_{\vec{n}^{\prime}, S^{\prime}}\left\langle\Delta_{S^{\prime}} \pi_{I}^{\prime}\left(\vec{n}^{\prime}, n_{t}\right) \Delta_{S} \pi_{I}^{\prime}\left(\vec{n}, n_{t}\right)\right\rangle: \rho_{S^{\prime}, I}^{a^{\dagger}, a}\left(\vec{n}^{\prime}\right) \rho_{S, I}^{a^{\dagger}, a}(\vec{n}) \rho^{a^{\dagger}, a}(\vec{n}):
$$

The three two-pion exchange terms $V_{\mathrm{TPE} 1}^{(3 N)}, V_{\mathrm{TPE} 2}^{(3 N)}, V_{\mathrm{TPE} 3}^{(3 N)}$ are

$$
\begin{aligned}
V_{\mathrm{TPE} 1}^{(3 N)} & =D_{\mathrm{TPE} 1} \frac{g_{A}^{2} \alpha_{t}^{2}}{4 f_{\pi}^{2} q_{\pi}^{2}} \sum_{\vec{n}, S, I} \sum_{\vec{n}^{\prime}, S^{\prime}} \sum_{\vec{n}^{\prime \prime}, S^{\prime \prime}}\left[\left\langle\Delta_{S^{\prime}} \pi_{I}^{\prime}\left(\vec{n}^{\prime}, n_{t}\right) \Delta_{S} \pi_{I}^{\prime}\left(\vec{n}, n_{t}\right)\right\rangle\right. \\
& \left.\times\left\langle\Delta_{S^{\prime \prime}} \pi_{I}^{\prime}\left(\vec{n}^{\prime \prime}, n_{t}\right) \Delta_{S} \pi_{I}^{\prime}\left(\vec{n}, n_{t}\right)\right\rangle: \rho_{S^{\prime}, I}^{a^{\dagger}, a}\left(\vec{n}^{\prime}\right) \rho_{S^{\prime \prime}, I}^{a^{\dagger}, a}\left(\vec{n}^{\prime \prime}\right) \rho^{a^{\dagger}, a}(\vec{n}):\right],
\end{aligned}
$$




$$
\begin{aligned}
& V_{\mathrm{TPE} 2}^{(3 N)}=D_{\mathrm{TPE} 2} m_{\pi}^{2} \frac{g_{A}^{2} \alpha_{t}^{2}}{4 f_{\pi}^{2} q_{\pi}^{2}} \sum_{\vec{n}, I} \sum_{\vec{n}^{\prime}, S^{\prime}} \sum_{\vec{n}^{\prime \prime}, S^{\prime \prime}}\left[\left\langle\Delta_{S^{\prime}} \pi_{I}^{\prime}\left(\vec{n}^{\prime}, n_{t}\right) \square \pi_{I}^{\prime}\left(\vec{n}, n_{t}\right)\right\rangle\right. \\
& \left.\times\left\langle\Delta_{S^{\prime \prime}} \pi_{I}^{\prime}\left(\vec{n}^{\prime \prime}, n_{t}\right) \square \pi_{I}^{\prime}\left(\vec{n}, n_{t}\right)\right\rangle: \rho_{S^{\prime}, I}^{a^{\dagger}, a}\left(\vec{n}^{\prime}\right) \rho_{S^{\prime \prime}, I}^{a^{\dagger}, a}\left(\vec{n}^{\prime \prime}\right) \rho^{a^{\dagger}, a}(\vec{n}):\right], \\
& V_{\mathrm{TPE} 3}^{(3 N)}=D_{\mathrm{TPE} 3} \frac{g_{A}^{2} \alpha_{t}^{2}}{4 f_{\pi}^{2} q_{\pi}^{2}} \sum_{\vec{n}, S_{1}, S_{2}, S_{3}} \sum_{I_{1}, I_{2}, I_{3}} \sum_{\vec{n}^{\prime}, S^{\prime}} \sum_{\vec{n}^{\prime \prime}, S^{\prime \prime}}[ \\
& \times\left\langle\Delta_{S^{\prime}} \pi_{I_{1}}^{\prime}\left(\vec{n}^{\prime}, n_{t}\right) \Delta_{S_{1}} \pi_{I_{1}}^{\prime}\left(\vec{n}, n_{t}\right)\right\rangle\left\langle\Delta_{S^{\prime \prime}} \pi_{I_{2}}^{\prime}\left(\vec{n}^{\prime \prime}, n_{t}\right) \Delta_{S_{2}} \pi_{I_{2}}^{\prime}\left(\vec{n}, n_{t}\right)\right\rangle \\
& \left.\times \varepsilon_{S_{1}, S_{2}, S_{3}} \varepsilon_{I_{1}, I_{2}, I_{3}}: \rho_{S^{\prime}, I_{1}}^{a^{\dagger}, a}\left(\vec{n}^{\prime}\right) \rho_{S^{\prime \prime}, I_{2}}^{a^{\dagger}, a}\left(\vec{n}^{\prime \prime}\right) \rho_{S_{3}, I_{3}}^{a^{\dagger}, a}(\vec{n}):\right] .
\end{aligned}
$$

Definitions for the $\square$ symbol and the two-point pion correlation functions are given in the Appendix.

In the continuum limit the tree-level scattering amplitudes are

$$
\begin{gathered}
\mathcal{A}\left[V_{\text {contact }}^{(3 N)}=D_{\text {contact }}\right. \\
\mathcal{A}\left[V_{\mathrm{OPE}}^{(3 N)}\right]=-D_{\mathrm{OPE}} \frac{g_{A}}{2 f_{\pi}} \sum_{P(A, B, C)} \frac{\vec{q}_{A} \cdot \vec{\sigma}_{A}}{q_{A}^{2}+m_{\pi}^{2}}\left(\vec{q}_{A} \cdot \vec{\sigma}_{B}\right)\left(\boldsymbol{\tau}_{A} \cdot \boldsymbol{\tau}_{B}\right) \\
\mathcal{A}\left[V_{\mathrm{TPE} 1}^{(3 N)}\right]=D_{\mathrm{TPE} 1} \frac{g_{A}^{2}}{4 f_{\pi}^{2}} \sum_{P(A, B, C)} \frac{\left(\vec{q}_{A} \cdot \vec{\sigma}_{A}\right)\left(\vec{q}_{B} \cdot \vec{\sigma}_{B}\right)}{\left(q_{A}^{2}+m_{\pi}^{2}\right)\left(q_{B}^{2}+m_{\pi}^{2}\right)}\left(\vec{q}_{A} \cdot \vec{q}_{B}\right)\left(\boldsymbol{\tau}_{A} \cdot \boldsymbol{\tau}_{B}\right) \\
\mathcal{A}\left[V_{\mathrm{TPE} 2}^{(3 N)}\right]=D_{\mathrm{TPE} 2} m_{\pi}^{2} \frac{g_{A}^{2}}{4 f_{\pi}^{2}} \sum_{P(A, B, C)} \frac{\left(\vec{q}_{A} \cdot \vec{\sigma}_{A}\right)\left(\vec{q}_{B} \cdot \vec{\sigma}_{B}\right)}{\left(q_{A}^{2}+m_{\pi}^{2}\right)\left(q_{B}^{2}+m_{\pi}^{2}\right)}\left(\boldsymbol{\tau}_{A} \cdot \boldsymbol{\tau}_{B}\right) \\
\mathcal{A}\left[V_{\mathrm{TPE} 3}^{(3 N)}=D_{\mathrm{TPE} 3} \frac{g_{A}^{2}}{4 f_{\pi}^{2}} \sum_{P(A, B, C)} \frac{\left(\vec{q}_{A} \cdot \vec{\sigma}_{A}\right)\left(\vec{q}_{B} \cdot \vec{\sigma}_{B}\right)}{\left(q_{A}^{2}+m_{\pi}^{2}\right)\left(q_{B}^{2}+m_{\pi}^{2}\right)}\left[\left(\vec{q}_{A} \times \vec{q}_{B}\right) \cdot \vec{\sigma}_{C}\right]\left[\left(\boldsymbol{\tau}_{A} \times \boldsymbol{\tau}_{B}\right) \cdot \boldsymbol{\tau}_{C}\right]\right.
\end{gathered}
$$

Comparing these with Eq. (12, 18$)$, we have

$$
\begin{gathered}
D_{\text {contact }}=-3 E=-\frac{3 c_{E}}{f_{\pi}^{4} \Lambda_{\chi}}, \quad D_{\mathrm{OPE}}=\frac{D}{4 f_{\pi}}=\frac{c_{D}}{4 f_{\pi}^{3} \Lambda_{\chi}}, \\
D_{\mathrm{TPE} 1}=\frac{c_{3}}{f_{\pi}^{2}}, \quad D_{\mathrm{TPE} 2}=-\frac{2 c_{1}}{f_{\pi}^{2}}, \quad D_{\mathrm{TPE} 3}=\frac{c_{4}}{2 f_{\pi}^{2}} .
\end{gathered}
$$




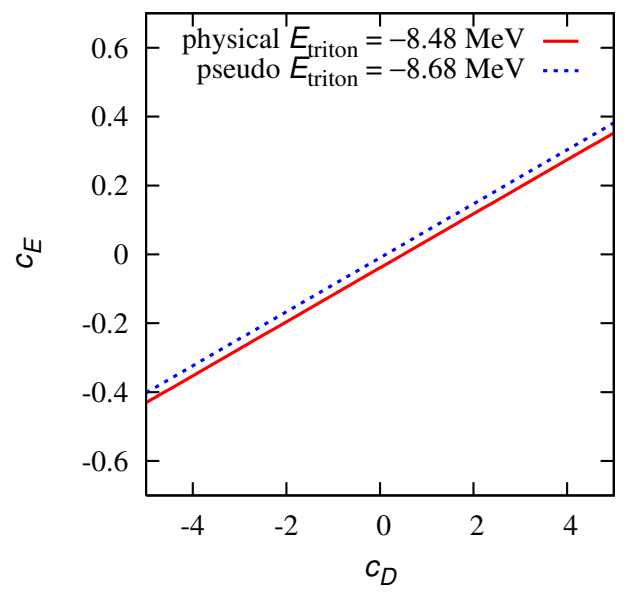

FIG. 3: Plot of $c_{E}$ versus $c_{D}$ when constrained by the triton energy at infinite volume. The solid line shows data matched to the physical triton energy, $-8.48 \mathrm{MeV}$. The dotted line shows results fitted to the pseudo triton energy, $-8.68 \mathrm{MeV}$, estimated by replacing $n n$ forces with $n p$ forces.

\section{A. Triton energy}

With the lattice transfer matrices $M_{\mathrm{LO}}, M_{\mathrm{NLO}}, M_{\mathrm{NNLO}}$, we use iterative sparse-matrix eigenvector methods to compute the triton energy for cubic periodic lattices. We consider cubes with side lengths $L=3,4,5,6,7,8$ and extract the infinite volume limit using the asymptotic parameterization [48],

$$
E_{\text {triton }}(L) \approx E_{\text {triton }}-\frac{C}{L} e^{-L / L_{0}}
$$

$L_{0}$ is a length scale associated with the physical size of the triton wavefunction. For the NNLO calculation we fix the coefficient $c_{E}$ as a function of $c_{D}$ by matching the physical triton energy at infinite volume, $-8.48 \mathrm{MeV}$. This constraint produces the solid line shown in Fig. 3. In the same figure the dotted line shows results obtained by fitting to the "pseudo" triton energy, $-8.68 \mathrm{MeV}$. This pseudo energy is an estimate of the triton energy when $n n$ interactions are replaced with $n p$ interactions [33]. This adjustment takes into account the systematic error in our isospin-symmetric calculations with two-nucleon interactions matched to $n p$ phase shifts. We note the similarity between Fig. 3 and other plots of $c_{E}$ versus $c_{D}$ found in Fig. 2 of Ref. [33] and Fig. 2 of Ref. [49]. In our case the plots are exactly linear due to our perturbative treatment of the three-nucleon interactions. In Fig. 4 we show $E_{\text {triton }}(L)$ versus $L$ measured in physical units at LO, NLO, and NNLO. For the NNLO 


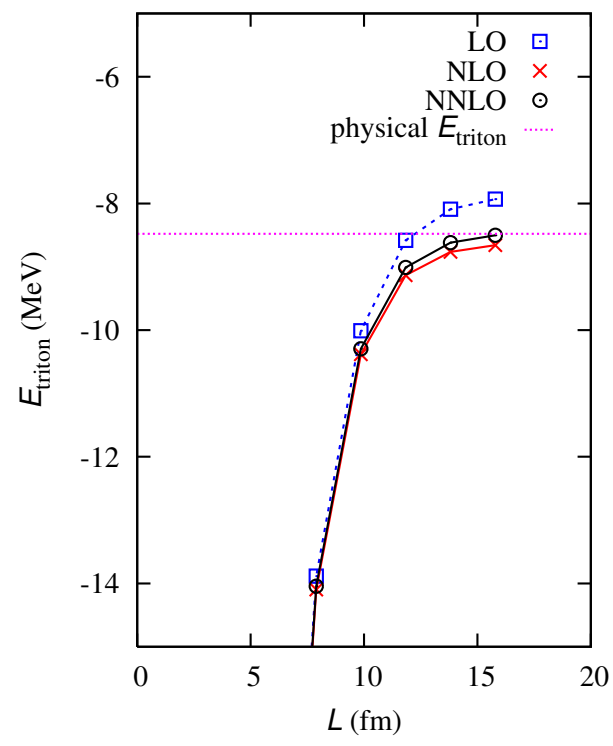

FIG. 4: Triton energy versus periodic lattice length at LO, NLO, and NNLO. For the NNLO results we show data for $c_{D}=1.0$ fitted to the physical triton energy.

calculation we show data for $c_{D}=1.0$ fitted to the physical triton energy. We see that the NLO and NNLO corrections each appear small enough to be treated using perturbation theory.

\section{B. Neutron-deuteron scattering}

Lüscher's formula relates the energy levels for a two-body system in a finite periodic cube to scattering phase shifts at infinite volume [50, 51]. We use this method to calculate neutron-deuteron scattering phase shifts in the spin-doublet and spin-quartet channels. For cubic lattice lengths $L=4,5,6,7,8$ we measure the three-nucleon energy levels relative to the threshold energy for a non-interacting neutron and deuteron in the same volume.

In Fig. 5 we plot $p \cot \delta$ versus $p^{2}$ in the center-of-mass frame for the spin-doublet channel. Using the pseudo $E_{\text {triton }}$ constraint for $c_{E}$, we show NNLO results for $c_{D}=-6.0,0.0,6.0$. This can be compared with the physical scattering length ${ }^{2} a_{n d}=-0.645 \pm 0.003_{\text {exp. }} \pm$ $0.007_{\text {th. }}$ fm [52] and pseudo scattering length ${ }^{2} a_{n d}=-0.45(4)$ fm resulting from adjusting the strength of $n n$ interactions to match $n p$ interactions [33]. A detailed discussion of isospin-breaking contributions to the neutron-deuteron scattering lengths can be found in 


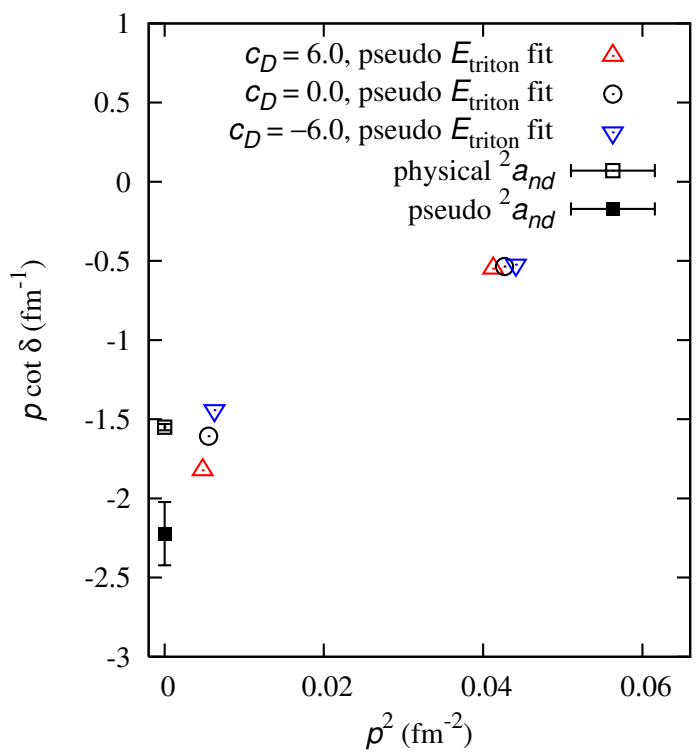

FIG. 5: NNLO results for spin-doublet neutron-deuteron scattering. We plot $p \cot \delta$ versus $p^{2}$ in the center-of-mass frame.

Ref. [53]. We match to the pseudo scattering length and find that $c_{D}$ lies in the range from -6.0 to +6.0 . This is a rather loose constraint since we already expect $c_{D} \sim O(1)$ based on the natural size of coefficients under renormalization group transformations. We consider alternative methods for constraining $c_{D}$ later in our discussion.

In Fig. 6] we plot $p \cot \delta$ for the spin-doublet channel for a wider range of $p^{2}$. For the NNLO calculation we show data for $c_{D}=1.0$ fitted to the physical value for $E_{\text {triton }}$. The experimental results are $n d$ and $p d$ scattering data from the partial wave analysis in Ref. [54]. The dashed line shows an empirical model introduced in Ref. [54] with a pole singularity in $p \cot \delta$ just below zero energy. This empirical model also accommodates data points for the triton and ${ }^{3} \mathrm{He}$ bound states at negative $p^{2}$. In our lattice data we also find non-trivial scattering behavior just below zero energy. The interpretation of these results and possible connections with the Efimov effect at finite volume are currently being studied [55, 56, 57]. The deuteron break-up threshold is near $p^{2}=0.07 \mathrm{fm}^{-2}$, and the agreement between lattice and experimental results for $n d$ scattering is quite good below break-up. Above the breakup threshold our analysis using Lüscher's finite volume formula does not take into account mixing between $n d$ and $n n p$ three-nucleon states. Therefore we expect significant errors in the case of strong mixing. 


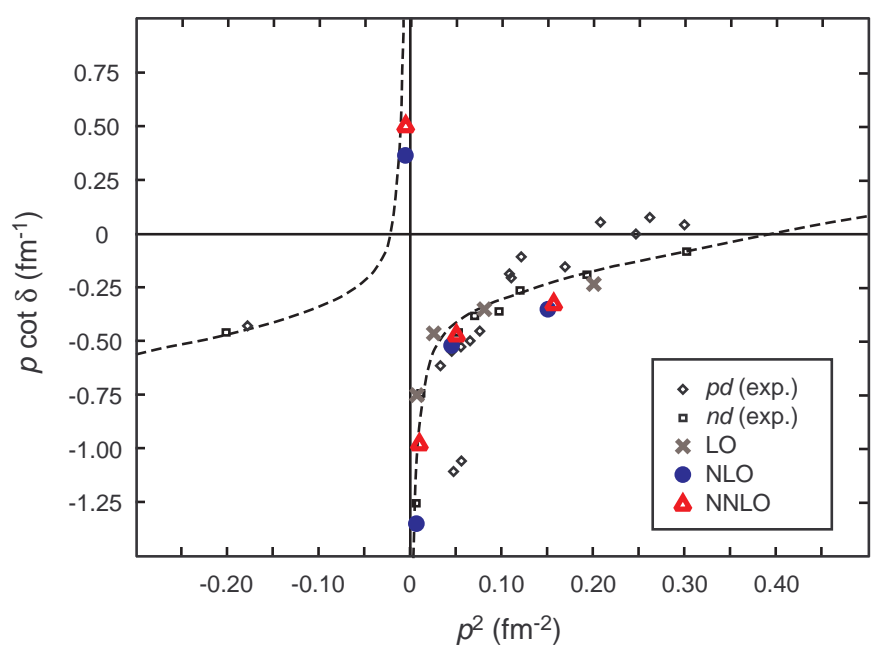

FIG. 6: Plot of $p \cot \delta$ versus $p^{2}$ for the spin-doublet channel in the center-of-mass frame. For the NNLO calculation we take $c_{D}=1.0$ fitted to the physical value for $E_{\text {triton }}$. The experimental results are from Ref. [54].

In Fig. 7 we plot $p \cot \delta$ for the spin-quartet channel. Again we show experimental results for $n d$ and $p d$ scattering from Ref. [54], and for the NNLO calculation we present data for $c_{D}=1.0$ fitted to the physical value for $E_{\text {triton }}$. The lattice data agree with experimental results for $n d$ scattering below deuteron break-up. However significant deviations appear above the break-up threshold. This may indicate mixing effects between $n d$ and nnp states.

\section{TRANSFER MATRICES WITH AUXILIARY FIELDS}

For systems with more than three nucleons, sparse-matrix calculations using the lattice transfer matrix are not practical at large volume. Instead we use projection Monte Carlo with auxiliary fields. A review of the auxiliary-field formalism can be found in Ref. [46]. We define $M^{\left(n_{t}\right)}\left(\pi_{I}^{\prime}, s, s_{I}\right)$ as the leading-order auxiliary-field transfer matrix at time step $n_{t}$,

$$
\begin{aligned}
M^{\left(n_{t}\right)}\left(\pi_{I}^{\prime}, s, s_{I}\right)=: & \exp \left\{-H_{\text {free }} \alpha_{t}-\frac{g_{A} \alpha_{t}}{2 f_{\pi} \sqrt{q_{\pi}}} \sum_{\vec{n}, S, I} \Delta_{S} \pi_{I}^{\prime}\left(\vec{n}, n_{t}\right) \rho_{S, I}^{a^{\dagger}, a}(\vec{n})\right. \\
& \left.+\sqrt{-C \alpha_{t}} \sum_{\vec{n}} s\left(\vec{n}, n_{t}\right) \rho^{a^{\dagger}, a}(\vec{n})+i \sqrt{C_{I^{2}} \alpha_{t}} \sum_{\vec{n}, I} s_{I}\left(\vec{n}, n_{t}\right) \rho_{I}^{a^{\dagger}, a}(\vec{n})\right\}:
\end{aligned}
$$




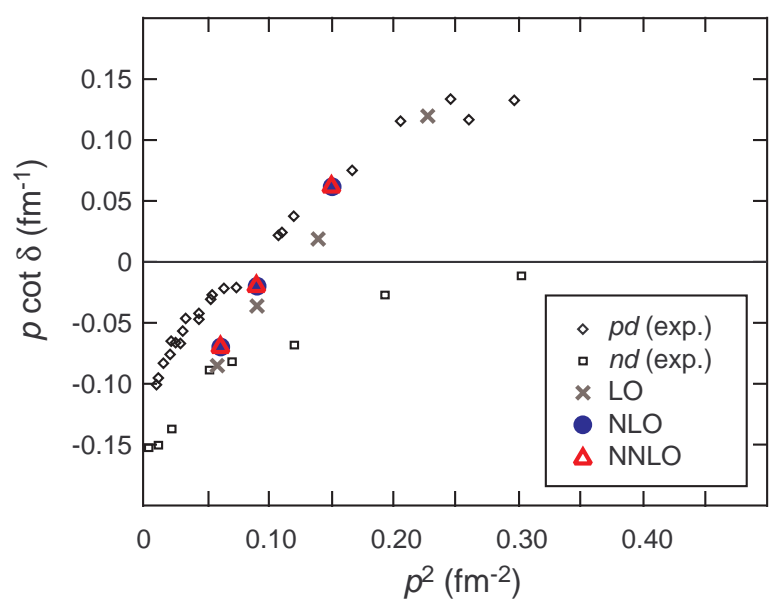

FIG. 7: Plot of $p \cot \delta$ versus $p^{2}$ for the spin-quartet channel in the center-of-mass frame. For the NNLO calculation we take $c_{D}=1.0$ fitted to the physical value for $E_{\text {triton }}$. The experimental results are from Ref. [54].

We can write $M_{\mathrm{LO}}$ as the normalized integral

$$
M_{\mathrm{LO}}=\frac{\int D \pi_{I}^{\prime} D s D s_{I} e^{-S_{\pi \pi}^{\left(n_{t}\right)}-S_{s s}^{\left(n_{t}\right)}} M^{\left(n_{t}\right)}\left(\pi_{I}^{\prime}, s, s_{I}\right)}{\int D \pi_{I}^{\prime} D s D s_{I} e^{-S_{\pi \pi}^{\left(n_{t}\right)}-S_{s s}^{\left(n_{t}\right)}}}
$$

where $S_{\pi \pi}^{\left(n_{t}\right)}$ is the piece of the instantaneous pion action at time step $n_{t}$,

$$
S_{\pi \pi}^{\left(n_{t}\right)}\left(\pi_{I}^{\prime}\right)=\frac{1}{2} \sum_{\vec{n}, I} \pi_{I}^{\prime}\left(\vec{n}, n_{t}\right) \pi_{I}^{\prime}\left(\vec{n}, n_{t}\right)-\frac{\alpha_{t}}{q_{\pi}} \sum_{\vec{n}, I, l} \pi_{I}^{\prime}\left(\vec{n}, n_{t}\right) \pi_{I}^{\prime}\left(\vec{n}+\hat{l}, n_{t}\right),
$$

and $S_{s s}^{\left(n_{t}\right)}$ is the auxiliary-field action at time step $n_{t}$,

$$
S_{s s}^{\left(n_{t}\right)}=\frac{1}{2} \sum_{\vec{n}, \vec{n}^{\prime}} s\left(\vec{n}, n_{t}\right) f^{-1}\left(\vec{n}-\vec{n}^{\prime}\right) s\left(\vec{n}^{\prime}, n_{t}\right)+\frac{1}{2} \sum_{I} \sum_{\vec{n}, \vec{n}^{\prime}} s_{I}\left(\vec{n}, n_{t}\right) f^{-1}\left(\vec{n}-\vec{n}^{\prime}\right) s_{I}\left(\vec{n}^{\prime}, n_{t}\right)
$$

with

$$
f^{-1}\left(\vec{n}-\vec{n}^{\prime}\right)=\frac{1}{L^{3}} \sum_{\vec{q}} \frac{1}{f(\vec{q})} e^{-i \vec{q} \cdot\left(\vec{n}-\vec{n}^{\prime}\right)}
$$


The NLO and NNLO interactions are treated using perturbation theory. We let

$$
\begin{aligned}
U^{\left(n_{t}\right)}(\varepsilon) & =\sum_{\vec{n}} \varepsilon_{\rho}\left(\vec{n}, n_{t}\right) \rho^{a^{\dagger}, a}(\vec{n})+\sum_{\vec{n}, S} \varepsilon_{\rho_{S}}\left(\vec{n}, n_{t}\right) \rho_{S}^{a^{\dagger}, a}(\vec{n})+\sum_{\vec{n}, S} \varepsilon_{\Delta_{S} \rho}\left(\vec{n}, n_{t}\right) \Delta_{S} \rho^{a^{\dagger}, a}(\vec{n}) \\
& +\sum_{\vec{n}, S, S^{\prime}} \varepsilon_{\Delta_{S} \rho_{S^{\prime}}}\left(\vec{n}, n_{t}\right) \Delta_{S} \rho_{S^{\prime}}^{a^{\dagger}, a}(\vec{n})+\sum_{\vec{n}, l} \varepsilon_{\nabla_{l}^{2} \rho}\left(\vec{n}, n_{t}\right) \nabla_{l}^{2} \rho^{a^{\dagger}, a}(\vec{n}) \\
& +\sum_{\vec{n}, l, S} \varepsilon_{\nabla_{l}^{2} \rho_{S}}\left(\vec{n}, n_{t}\right) \nabla_{l}^{2} \rho_{S}^{a^{\dagger}, a}(\vec{n})+\sum_{\vec{n}, l} \varepsilon_{\Pi_{l}}\left(\vec{n}, n_{t}\right) \Pi_{l}^{a^{\dagger}, a}(\vec{n})+\sum_{\vec{n}, l, S} \varepsilon_{\Pi_{l, S}}\left(\vec{n}, n_{t}\right) \Pi_{l, S}^{a^{\dagger}, a}(\vec{n}),
\end{aligned}
$$

and

$$
\begin{aligned}
U_{I^{2}}^{\left(n_{t}\right)}(\varepsilon) & =\sum_{\vec{n}, I} \varepsilon_{\rho_{I}}\left(\vec{n}, n_{t}\right) \rho_{I}^{a^{\dagger}, a}(\vec{n})+\sum_{\vec{n}, S, I} \varepsilon_{\rho_{S, I}}\left(\vec{n}, n_{t}\right) \rho_{S, I}^{a^{\dagger}, a}(\vec{n})+\sum_{\vec{n}, S, I} \varepsilon_{\Delta_{S} \rho_{I}}\left(\vec{n}, n_{t}\right) \Delta_{S} \rho_{I}^{a^{\dagger}, a}(\vec{n}) \\
& +\sum_{\vec{n}, S, S^{\prime}, I} \varepsilon_{\Delta_{S} \rho_{S^{\prime}, I}}\left(\vec{n}, n_{t}\right) \Delta_{S} \rho_{S^{\prime}, I}^{a^{\dagger}, a}(\vec{n})+\sum_{\vec{n}, l, I} \varepsilon_{\nabla_{l}^{2} \rho_{I}}\left(\vec{n}, n_{t}\right) \nabla_{l}^{2} \rho_{I}^{a^{\dagger}, a}(\vec{n}) \\
& +\sum_{\vec{n}, l, S, I} \varepsilon_{\nabla_{l}^{2} \rho_{S, I}}\left(\vec{n}, n_{t}\right) \nabla_{l}^{2} \rho_{S, I}^{a^{\dagger}, a}(\vec{n})+\sum_{\vec{n}, l, I} \varepsilon_{\Pi_{l, I}}\left(\vec{n}, n_{t}\right) \Pi_{l, I}^{a^{\dagger}, a}(\vec{n})+\sum_{\vec{n}, l, S, I} \varepsilon_{\Pi_{l, S, I}}\left(\vec{n}, n_{t}\right) \Pi_{l, S, I}^{a^{\dagger}, a}(\vec{n}) .
\end{aligned}
$$

With these extra fields and linear functionals we define

$$
\begin{aligned}
& M^{\left(n_{t}\right)}\left(\pi_{I}^{\prime}, s, s_{I}, \varepsilon\right) \\
& =: \exp \left\{-H_{\text {free }} \alpha_{t}-\frac{g_{A} \alpha_{t}}{2 f_{\pi} \sqrt{q_{\pi}}} \sum_{\vec{n}, S, I} \Delta_{S} \pi_{I}^{\prime}\left(\vec{n}, n_{t}\right) \rho_{S, I}^{a^{\dagger}, a}(\vec{n})\right. \\
& \left.+\sqrt{-C \alpha_{t}} \sum_{\vec{n}} s\left(\vec{n}, n_{t}\right) \rho^{a^{\dagger}, a}(\vec{n})+i \sqrt{C_{I^{2}} \alpha_{t}} \sum_{\vec{n}, I} s_{I}\left(\vec{n}, n_{t}\right) \rho_{I}^{a^{\dagger}, a}(\vec{n})+U^{\left(n_{t}\right)}(\varepsilon)+U_{I^{2}}^{\left(n_{t}\right)}(\varepsilon)\right\}:
\end{aligned}
$$

In Ref. [6, 7, 46] there were factors of $\sqrt{\alpha_{t}}$ multiplying $U^{\left(n_{t}\right)}(\varepsilon)$ and $U_{I^{2}}^{\left(n_{t}\right)}(\varepsilon)$. We have removed these factors here as they complicate the discussion of the three-body interactions at NNLO. Let $M^{\left(n_{t}\right)}(\varepsilon)$ be the normalized integral,

$$
M^{\left(n_{t}\right)}(\varepsilon)=\frac{\int D \pi^{\prime} D s D s_{I} e^{-S_{\pi \pi}^{\left(n_{t}\right)}-S_{s s}^{\left(n_{t}\right)}} M^{\left(n_{t}\right)}\left(\pi_{I}^{\prime}, s, s_{I}, \varepsilon\right)}{\int D \pi^{\prime} D s D s_{I} e^{-S_{\pi \pi}^{\left(n_{t}\right)}-S_{s s}^{\left(n_{t}\right)}}} .
$$

When all $\varepsilon$ fields are set to zero we recover $M_{\mathrm{LO}}$,

$$
M^{\left(n_{t}\right)}(0)=M_{\mathrm{LO}}
$$


To first order in perturbation theory the NLO interactions in $M_{\mathrm{NLO}}$ can be written as a sum of bilinear derivatives of $M^{\left(n_{t}\right)}(\varepsilon)$ with respect to the $\varepsilon$ fields at $\varepsilon=0$,

$$
\begin{aligned}
M_{\mathrm{NLO}} & =M_{\mathrm{LO}} \\
& -\left.\frac{1}{2} \Delta C \alpha_{t} \sum_{\vec{n}} \frac{\delta}{\delta \varepsilon_{\rho}\left(\vec{n}, n_{t}\right)} \frac{\delta}{\delta \varepsilon_{\rho}\left(\vec{n}, n_{t}\right)} M^{\left(n_{t}\right)}(\varepsilon)\right|_{\varepsilon=0} \\
& +\left.\frac{1}{2} C_{q^{2}} \alpha_{t} \sum_{\vec{n}} \frac{\delta}{\delta \varepsilon_{\rho}\left(\vec{n}, n_{t}\right)} \frac{\delta}{\delta \varepsilon_{\nabla_{l}^{2} \rho}\left(\vec{n}, n_{t}\right)} M^{\left(n_{t}\right)}(\varepsilon)\right|_{\varepsilon=0}+\cdots
\end{aligned}
$$

Similarly for NNLO we have

$$
M_{\mathrm{NNLO}}=M_{\mathrm{NLO}}+\frac{\int D \pi^{\prime} D s D s_{I} e^{-S_{\pi \pi}^{\left(n_{t}\right)}-S_{s s}^{\left(n_{t}\right)}} \Delta M_{\mathrm{NNLO}}^{\left(n_{t}\right)}\left(\pi^{\prime}\right)}{\int D \pi^{\prime} D s D s_{I} e^{-S_{\pi \pi}^{\left(n_{t}\right)}-S_{s s}^{\left(n_{t}\right)}}},
$$

where

$$
\begin{aligned}
\Delta M_{\mathrm{NNLO}}^{\left(n_{t}\right)}\left(\pi^{\prime}\right) & =M_{\mathrm{contact}}^{(3 N)\left(n_{t}\right)}\left(\pi^{\prime}\right)+M_{\mathrm{OPE}}^{(3 N)\left(n_{t}\right)}\left(\pi^{\prime}\right) \\
& +M_{\mathrm{TPE} 1}^{(3 N)\left(n_{t}\right)}\left(\pi^{\prime}\right)+M_{\mathrm{TPE} 2}^{(3 N)\left(n_{t}\right)}\left(\pi^{\prime}\right)+M_{\mathrm{TPE} 3}^{(3 N)\left(n_{t}\right)}\left(\pi^{\prime}\right) .
\end{aligned}
$$

The three-nucleon contact interaction is

$$
M_{\text {contact }}^{(3 N)\left(n_{t}\right)}\left(\pi^{\prime}\right)=-\left.\frac{1}{6} D_{\text {contact }} \alpha_{t} \sum_{\vec{n}}\left[\frac{\delta}{\delta \varepsilon_{\rho}\left(\vec{n}, n_{t}\right)}\right]^{3} M^{\left(n_{t}\right)}(\varepsilon)\right|_{\varepsilon=0},
$$

and the one-pion exchange interaction has the form

$$
M_{\mathrm{OPE}}^{(3 N)\left(n_{t}\right)}\left(\pi^{\prime}\right)=-\left.D_{\mathrm{OPE}} \frac{\alpha_{t}}{\sqrt{q_{\pi}}} \sum_{\vec{n}, S, I} \Delta_{S} \pi_{I}^{\prime}\left(\vec{n}, n_{t}\right) \frac{\delta}{\delta \varepsilon_{\rho_{S, I}}\left(\vec{n}, n_{t}\right)} \frac{\delta}{\delta \varepsilon_{\rho}\left(\vec{n}, n_{t}\right)} M^{\left(n_{t}\right)}(\varepsilon)\right|_{\varepsilon=0} .
$$

The three two-pion exchange terms are

$$
\begin{aligned}
& M_{\mathrm{TPE} 1}^{(3 N)\left(n_{t}\right)}\left(\pi^{\prime}\right)=-D_{\mathrm{TPE} 1} \frac{\alpha_{t}}{q_{\pi}} \\
& \times\left.\sum_{\vec{n}, S, I}\left[\Delta_{S} \pi_{I}^{\prime}\left(\vec{n}, n_{t}\right) \Delta_{S} \pi_{I}^{\prime}\left(\vec{n}, n_{t}\right)-\left\langle\Delta_{S} \pi_{I}^{\prime}\left(\vec{n}, n_{t}\right) \Delta_{S} \pi_{I}^{\prime}\left(\vec{n}, n_{t}\right)\right\rangle\right] \frac{\delta M^{\left(n_{t}\right)}(\varepsilon)}{\delta \varepsilon_{\rho}\left(\vec{n}, n_{t}\right)}\right|_{\varepsilon=0}, \\
& \quad M_{\mathrm{TPE} 2}^{(3 N)\left(n_{t}\right)}\left(\pi^{\prime}\right)=-D_{\mathrm{TPE} 2} \frac{m_{\pi}^{2} \alpha_{t}}{q_{\pi}} \\
& \quad \times\left.\sum_{\vec{n}, I}\left[\square \pi_{I}^{\prime}\left(\vec{n}, n_{t}\right) \square \pi_{I}^{\prime}\left(\vec{n}, n_{t}\right)-\left\langle\square \pi_{I}^{\prime}\left(\vec{n}, n_{t}\right) \square \pi_{I}^{\prime}\left(\vec{n}, n_{t}\right)\right\rangle\right] \frac{\delta M^{\left(n_{t}\right)}(\varepsilon)}{\delta \varepsilon_{\rho}\left(\vec{n}, n_{t}\right)}\right|_{\varepsilon=0},
\end{aligned}
$$




$$
\begin{aligned}
& M_{\mathrm{TPE} 3}^{(3 N)\left(n_{t}\right)}\left(\pi^{\prime}\right)=-D_{\mathrm{TPE} 3} \frac{\alpha_{t}}{q_{\pi}} \\
& \times\left.\sum_{\vec{n}, S_{1}, S_{2}, S_{3}} \sum_{I_{1}, I_{2}, I_{3}} \varepsilon_{S_{1}, S_{2}, S_{3}} \varepsilon_{I_{1}, I_{2}, I_{3}} \Delta_{S_{1}} \pi_{I_{1}}^{\prime}\left(\vec{n}, n_{t}\right) \Delta_{S_{2}} \pi_{I_{2}}^{\prime}\left(\vec{n}, n_{t}\right) \frac{\delta M^{\left(n_{t}\right)}(\varepsilon)}{\delta \varepsilon_{\rho_{S_{3}, I_{3}}}\left(\vec{n}, n_{t}\right)}\right|_{\varepsilon=0} .
\end{aligned}
$$

We extract the properties of the ground state using Euclidean-time projection. Let $\left|\Psi^{\text {free }}\right\rangle$ be a Slater determinant of free-particle standing waves on the lattice. We construct the trial state $\left|\Psi\left(t^{\prime}\right)\right\rangle$ using

$$
\left|\Psi\left(t^{\prime}\right)\right\rangle=\left(M_{\mathrm{SU}(4) \not x^{\prime}}\right)^{L_{t_{o}}}\left|\Psi^{\text {free }}\right\rangle
$$

where $t^{\prime}=L_{t_{o}} \alpha_{t}$ and $L_{t_{o}}$ is the number of "outer" time steps. As the notation suggests, the transfer matrix $M_{\mathrm{SU}(4) \not t}$ is invariant under an exact Wigner $\mathrm{SU}(4)$ symmetry and acts as an approximate low-energy filter. The amplitude $Z(t)$ is defined as

$$
Z(t)=\left\langle\Psi\left(t^{\prime}\right)\left|\left(M_{\mathrm{LO}}\right)^{L_{t_{i}}}\right| \Psi\left(t^{\prime}\right)\right\rangle
$$

where $t=L_{t_{i}} \alpha_{t}$ and $L_{t_{i}}$ is the number of "inner" time steps. The transient energy

$$
E_{\mathrm{LO}}\left(t+\alpha_{t} / 2\right)
$$

is given by the ratio of the amplitudes for $t$ and $t+\alpha_{t}$,

$$
e^{-E_{\mathrm{LO}}\left(t+\alpha_{t} / 2\right) \cdot \alpha_{t}}=\frac{Z\left(t+\alpha_{t}\right)}{Z(t)} .
$$

The ground state energy $E_{0, \mathrm{LO}}$ equals the asymptotic limit,

$$
E_{0, \mathrm{LO}}=\lim _{t \rightarrow \infty} E_{\mathrm{LO}}\left(t+\alpha_{t} / 2\right)
$$

We calculate these Euclidean-time projection amplitudes using auxiliary fields. For a given configuration of auxiliary and pion fields, the contribution to the amplitude $Z(t)$ is proportional to the determinant of an $A \times A$ matrix of one-body amplitudes where $A$ is the number of nucleons. Integrations over auxiliary and pion field configurations are computed using hybrid Monte Carlo. Details of the method can be found in Ref. [4, 46, 58, 59].

For the ground state energy at NLO and NNLO we compute expectation values of $M_{\mathrm{LO}}$, $M_{\mathrm{NLO}}, M_{\mathrm{NNLO}}$ inserted in the middle of a string of $M_{\mathrm{LO}}$ transfer matrices,

$$
Z_{M_{\mathrm{LO}}}(t)=\left\langle\Psi\left(t^{\prime}\right)\left|\left(M_{\mathrm{LO}}\right)^{L_{t_{i}} / 2} M_{\mathrm{LO}}\left(M_{\mathrm{LO}}\right)^{L_{t_{i}} / 2}\right| \Psi\left(t^{\prime}\right)\right\rangle
$$




$$
\begin{aligned}
Z_{M_{\mathrm{NLO}}}(t) & =\left\langle\Psi\left(t^{\prime}\right)\left|\left(M_{\mathrm{LO}}\right)^{L_{t_{i}} / 2} M_{\mathrm{NLO}}\left(M_{\mathrm{LO}}\right)^{L_{t_{i}} / 2}\right| \Psi\left(t^{\prime}\right)\right\rangle, \\
Z_{M_{\mathrm{NNLO}}}(t) & =\left\langle\Psi\left(t^{\prime}\right)\left|\left(M_{\mathrm{LO}}\right)^{L_{t_{i}} / 2} M_{\mathrm{NNLO}}\left(M_{\mathrm{LO}}\right)^{L_{t_{i}} / 2}\right| \Psi\left(t^{\prime}\right)\right\rangle .
\end{aligned}
$$

From the ratio of amplitudes,

$$
\frac{Z_{M_{\mathrm{NLO}}}(t)}{Z_{M_{\mathrm{LO}}}(t)}=1-\Delta E_{\mathrm{NLO}}(t) \alpha_{t}+\cdots,
$$

we define the transient NLO energy correction $\Delta E_{\mathrm{NLO}}(t)$. The ellipsis denotes terms which are beyond first order in the NLO coefficients. The NLO ground state energy $E_{0, \mathrm{NLO}}$ is calculated using

$$
E_{0, \mathrm{NLO}}=E_{0, \mathrm{LO}}+\lim _{t \rightarrow \infty} \Delta E_{\mathrm{NLO}}(t)
$$

Similarly at NNLO we have

$$
\frac{Z_{M_{\mathrm{NNLO}}}(t)}{Z_{M_{\mathrm{LO}}}(t)}=1-\Delta E_{\mathrm{NNLO}}(t) \alpha_{t}+\cdots,
$$

and

$$
E_{0, \mathrm{NNLO}}=E_{0, \mathrm{NLO}}+\lim _{t \rightarrow \infty} \Delta E_{\mathrm{NNLO}}(t)
$$

\section{PRECISION TESTS OF MONTE CARLO SIMULATIONS}

We use the three-nucleon system to test the auxiliary-field Monte Carlo simulations. The same observables are calculated using both auxiliary-field Monte Carlo and the exact transfer matrix without auxiliary fields. We choose a small system so that stochastic errors are small enough to expose disagreement at the $0.1 \%-1 \%$ level. We choose the spatial length of the lattice to be $L=3$ and set the outer time steps $L_{t_{o}}=0$ and inner time steps $L_{t_{i}}=4$. With 2048 processors we generate a total of about $10^{8}$ hybrid Monte Carlo trajectories. Each processor runs completely independent trajectories, and we compute averages and stochastic errors by comparing the results of all processors.

We choose $\left|\Psi^{\text {free }}\right\rangle$ to be a spin-doublet isospin-doublet state built from the Slater determinant of standing waves $\left|\psi_{1}\right\rangle,\left|\psi_{2}\right\rangle,\left|\psi_{3}\right\rangle$ with

$$
\left\langle 0\left|a_{i, j}(\vec{n})\right| \psi_{1}\right\rangle \propto \delta_{i, 0} \delta_{j, 1}, \quad\left\langle 0\left|a_{i, j}(\vec{n})\right| \psi_{2}\right\rangle \propto \delta_{i, 1} \delta_{j, 1}, \quad\left\langle 0\left|a_{i, j}(\vec{n})\right| \psi_{3}\right\rangle \propto \delta_{i, 0} \delta_{j, 0} .
$$

In Table \w whow Monte Carlo results for the energy (MC) versus exact transfer matrix calculations (Exact) at LO, NLO, and NNLO. The NNLO data uses $c_{D}=1.0$ with $c_{E}$ 
TABLE II: Monte Carlo results for the energy versus exact transfer matrix calculations

\begin{tabular}{||c|c|c||}
\hline \hline Energies & $\mathrm{MC}$ & Exact \\
\hline$E_{\mathrm{LO}}\left(t+\alpha_{t} / 2\right)[\mathrm{MeV}]$ & $-48.873(18)$ & -48.8823 \\
\hline$\Delta E_{\mathrm{NLO}}(t)[\mathrm{MeV}]$ & $0.5509(8)$ & 0.55100 \\
\hline$\Delta E_{\mathrm{NNLO}}(t)[\mathrm{MeV}]$ & $-0.967(3)$ & -0.96718 \\
\hline \hline
\end{tabular}

TABLE III: Monte Carlo results versus exact transfer matrix calculations for the derivative of the energy with respect to NLO coefficients

\begin{tabular}{||c|c|c||}
\hline \hline NLO energy derivatives & $\mathrm{MC}$ & Exact \\
\hline$\frac{\partial\left(\Delta E_{\mathrm{NLO}}(t)\right)}{\partial(\Delta C)}\left[10^{4} \mathrm{MeV}^{3}\right]$ & $3.9037(12)$ & 3.90226 \\
\hline$\frac{\partial\left(\Delta E_{\mathrm{NLO}}(t)\right)}{\partial\left(\Delta C_{I^{2}}\right)}\left[10^{4} \mathrm{MeV}^{3}\right]$ & $-4.847(2)$ & -4.84331 \\
\hline$\frac{\partial\left(\Delta E_{\mathrm{NLO}}(t)\right)}{\partial\left(C_{q 2}\right)}\left[10^{9} \mathrm{MeV}^{5}\right]$ & $-2.0105(6)$ & -2.01059 \\
\hline$\frac{\partial\left(\Delta E_{\mathrm{NLO}}(t)\right)}{\partial\left(C_{I^{2}, q^{2}}\right)}\left[10^{9} \mathrm{MeV}^{5}\right]$ & $2.9230(14)$ & 2.92424 \\
\hline$\frac{\partial\left(\Delta E_{\mathrm{NLO}}(t)\right)}{\partial\left(C_{S^{2}, q^{2}}\right)}\left[10^{9} \mathrm{MeV}^{5}\right]$ & $0.1860(12)$ & 0.18411 \\
\hline$\frac{\partial\left(\Delta E_{\mathrm{NLO}}(t)\right)}{\partial\left(C_{S^{2}, I^{2}, q^{2}}\right)}\left[10^{9} \mathrm{MeV}^{5}\right]$ & $5.094(2)$ & 5.09371 \\
\hline$\frac{\partial\left(\Delta E_{\mathrm{NLO}}(t)\right)}{\partial\left(C_{(q \cdot S)^{2}}\right)}\left[10^{9} \mathrm{MeV}^{5}\right]$ & $-1.5892(3)$ & -1.58898 \\
\hline$\frac{\partial\left(\Delta E_{\mathrm{NLO}}(t)\right)}{\partial\left(C_{I^{2},(q \cdot S)^{2}}\right)}\left[10^{9} \mathrm{MeV}^{5}\right]$ & $6.8019(11)$ & 6.80197 \\
\hline$\frac{\partial\left(\Delta E_{\mathrm{NLO}}(t)\right)}{\partial\left(C_{(i q \times S) \cdot k}\right)}\left[10^{9} \mathrm{MeV}^{5}\right]$ & $0.3417(2)$ & 0.34164 \\
\hline$\frac{\partial\left(\Delta E_{\mathrm{NLO}}(t)\right)}{\partial\left(C_{I^{2},(i q \times S) \cdot k}\right)}\left[10^{9} \mathrm{MeV}^{5}\right]$ & $-1.0092(5)$ & -1.00932 \\
\hline \hline
\end{tabular}

fitted to the physical triton energy. In Table III we compare Monte Carlo results with exact transfer matrix calculations for the derivative of the energy with respect to each NLO coefficient. In Table [V] we make the same comparison for the derivative of the energy with respect to each NNLO coefficient. The numbers in parentheses are the estimated stochastic errors. In all cases the agreement between Monte Carlo results and exact transfer calculations is consistent with estimated stochastic errors. 
TABLE IV: Monte Carlo results versus exact transfer matrix calculations for the derivative of the energy with respect to NNLO coefficients

\begin{tabular}{||c|c|c||}
\hline \hline NNLO energy derivatives & $\mathrm{MC}$ & Exact \\
\hline$\frac{\partial\left(\Delta E_{\mathrm{NNLO}}(t)\right)}{\partial\left(D_{\mathrm{contact}}\right)}\left[10^{8} \mathrm{MeV}^{6}\right]$ & $1.162(4)$ & 1.1609 \\
\hline$\frac{\partial\left(\Delta E_{\mathrm{NNLO}}(t)\right)}{\partial\left(D_{\mathrm{OPE}}\right)}\left[10^{7} \mathrm{MeV}^{5}\right]$ & $-5.858(6)$ & -5.8623 \\
\hline$\frac{\partial\left(\Delta E_{\mathrm{NNLO}}(t)\right)}{\partial\left(D_{\mathrm{TPE} 1}\right)}\left[10^{5} \mathrm{MeV}^{4}\right]$ & $14.46(5)$ & 14.468 \\
\hline$\frac{\partial\left(\Delta E_{\mathrm{NNLO}}(t)\right)}{\partial\left(D_{\mathrm{TPE} 2}\right)}\left[10^{5} \mathrm{MeV}^{4}\right]$ & $2.24(3)$ & 2.2595 \\
\hline$\frac{\partial\left(\Delta E_{\mathrm{NNLO}}(t)\right)}{\partial\left(D_{\mathrm{TPE} 3}\right)}\left[10^{5} \mathrm{MeV}^{4}\right]$ & $-10.02(6)$ & -10.022 \\
\hline \hline
\end{tabular}

\section{ENERGY OF THE $\alpha$ PARTICLE}

We simulate the $\alpha$ particle on cubic periodic lattices with length $L=5,6,7,8$. These correspond with physical lengths $L=9.9,11.8,13.8,15.8 \mathrm{fm}$. For $\left|\Psi^{\text {free }}\right\rangle$ we take the Slater determinant formed by standing waves

$$
\begin{aligned}
& \left\langle 0\left|a_{i, j}(\vec{n})\right| \psi_{1}\right\rangle \propto \delta_{i, 0} \delta_{j, 1}, \quad\left\langle 0\left|a_{i, j}(\vec{n})\right| \psi_{2}\right\rangle \propto \delta_{i, 1} \delta_{j, 1}, \\
& \left\langle 0\left|a_{i, j}(\vec{n})\right| \psi_{3}\right\rangle \propto \delta_{i, 0} \delta_{j, 0}, \quad\left\langle 0\left|a_{i, j}(\vec{n})\right| \psi_{4}\right\rangle \propto \delta_{i, 1} \delta_{j, 0} .
\end{aligned}
$$

For each value of $L_{t_{i}}$ a total of about $6 \times 10^{6}$ hybrid Monte Carlo trajectories are generated by 2048 processors, each running completely independent trajectories. Averages and stochastic errors are computed by comparing the results of all processors.

For $L=5,6,7,8$ we show Monte Carlo results in Fig. 8 for

$$
E_{\mathrm{LO}}(t), \quad E_{\mathrm{LO}}(t)+\Delta E_{\mathrm{NLO}}(t), \quad E_{\mathrm{LO}}(t)+\Delta E_{\mathrm{NLO}}(t)+\Delta E_{\mathrm{NNLO}}(t),
$$

versus Euclidean time $t$. These are labelled as LO, NLO, and NNLO respectively. The NNLO data uses $c_{D}=1.0$ with $c_{E}$ fitted to the physical triton energy. In addition to the Monte Carlo data we plot the asymptotic forms for each term in Eq. (91) using

$$
\begin{gathered}
E_{\mathrm{LO}}(t) \approx E_{0, \mathrm{LO}}+A_{\mathrm{LO}} e^{-\delta E \cdot t}, \\
\Delta E_{\mathrm{NLO}}(t) \approx E_{0, \mathrm{NLO}}-E_{0, \mathrm{LO}}+B_{\mathrm{NLO}} e^{-\delta E \cdot t / 2}, \\
\Delta E_{\mathrm{NNLO}}(t) \approx E_{0, \mathrm{NNLO}}-E_{0, \mathrm{NLO}}+B_{\mathrm{NNLO}} e^{-\delta E \cdot t / 2} .
\end{gathered}
$$




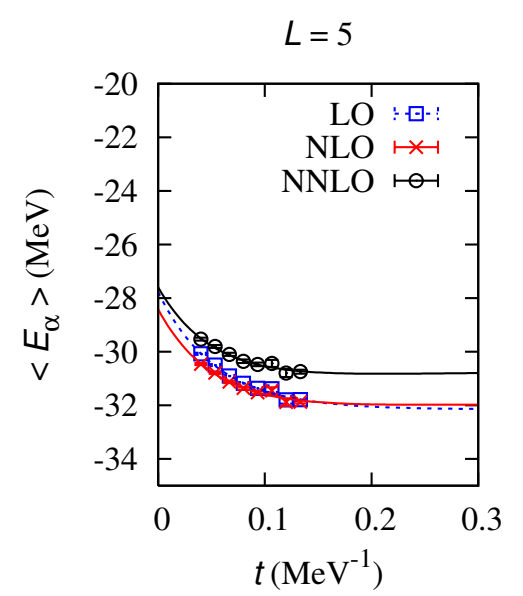

(a)

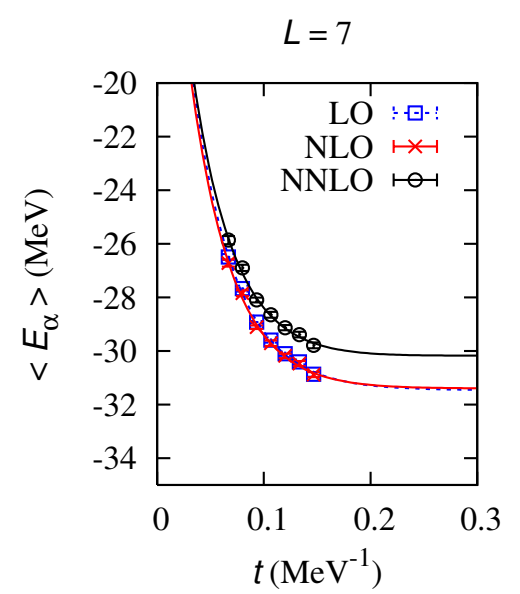

(c)

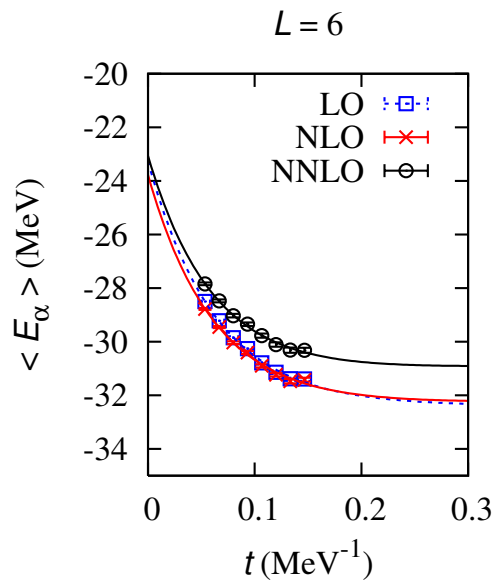

(b)

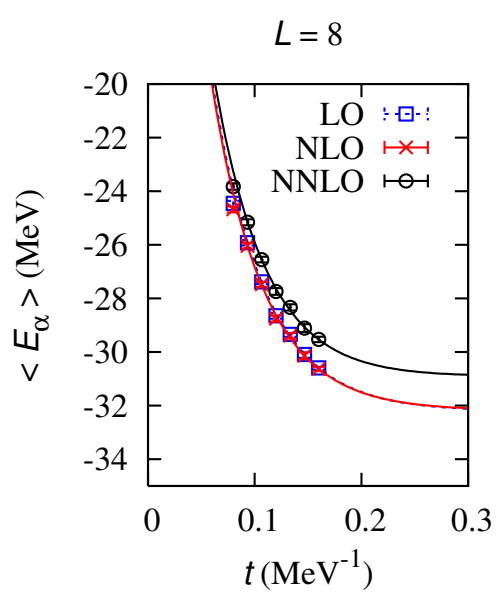

(d)

FIG. 8: Monte Carlo results for $\alpha$-particle energies versus Euclidean time $t$ at LO, NLO, and NNLO. We also plot fitted asymptotic expressions. The NNLO data uses $c_{D}=1.0$ with $c_{E}$ fitted to the physical triton energy.

The unknown coefficients $A_{\mathrm{LO}}, B_{\mathrm{NLO}}, B_{\mathrm{NNLO}}$, and energy gap $\delta E$, are determined by least squares fitting. The $e^{-\delta E \cdot t}$ dependence in Eq. (92) comes from the contribution of low-energy excitations with energy gap $\delta E$ above the $\alpha$ particle. The $e^{-\delta E \cdot t / 2}$ dependence in Eq. (93) is due to matrix elements of $M_{\mathrm{NLO}}$ between the $\alpha$ particle and low-energy excitations at $\delta E$. Similarly the $e^{-\delta E \cdot t / 2}$ dependence in Eq. (94) is from matrix elements of $M_{\mathrm{NNLO}}$. The chi-squared per degree of freedom for the fits are 1.5 for $L=5,1.0$ for $L=6,0.7$ for $L=7$, and 0.6 for $L=8$. The small relative size of the NLO and NNLO energy corrections suggest that the perturbative treatment of these terms appears reasonable. 


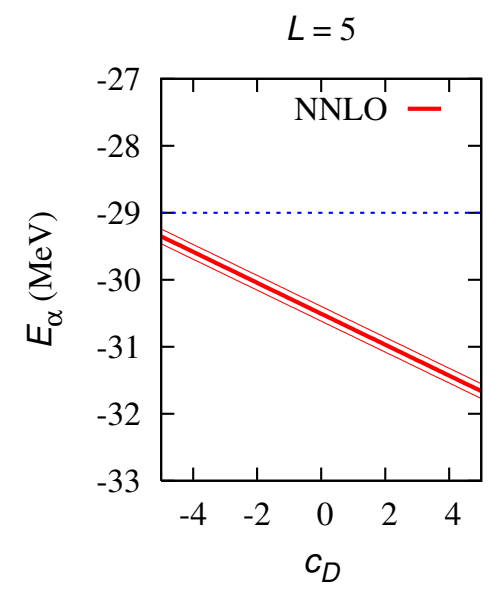

(a)

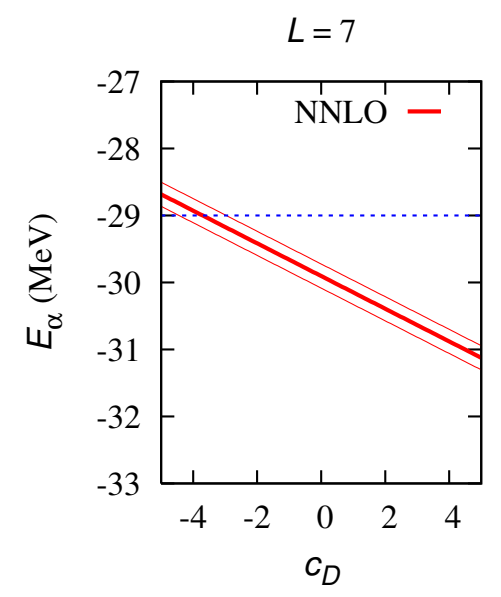

(c)

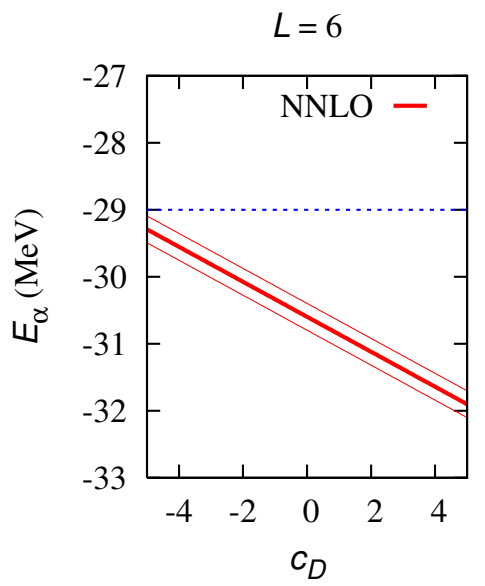

(b)

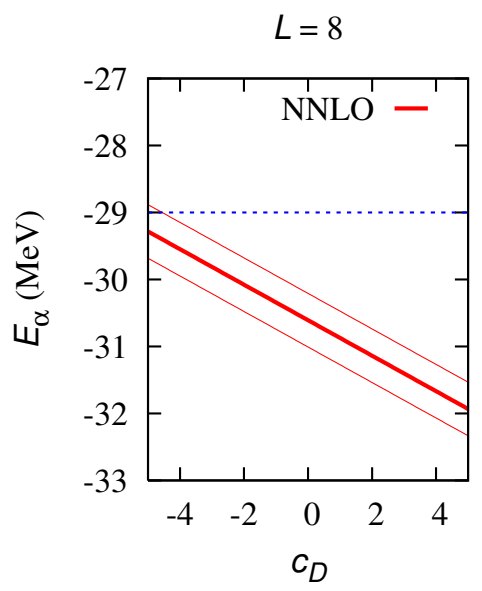

(d)

FIG. 9: Energy of the $\alpha$ particle at NNLO versus $c_{D}$. The contact interaction $c_{E}$ is fitted to the physical triton energy. The dotted line is the estimated Coulomb-subtracted energy $-29.0 \mathrm{MeV}$.

In Fig. 9 we plot the NNLO $\alpha$-particle energy versus $c_{D}$, with $c_{E}$ fitted to the physical triton energy. The bands indicate the estimated error due to stochastic noise and asymptotic fits at large $t$. The $\alpha$ energy should approach the infinite volume limit from below, similar to our results for the triton energy. Hence the deviation between data at $L=7$ and $L=8$ is likely due to stochastic noise and fit errors rather than finite volume effects. The $\alpha$ energy shown at $-29.0 \mathrm{MeV}$ is the estimated Coulomb-subtracted energy [30]. At large volumes the best agreement with the Coulomb-subtracted $\alpha$ energy occurs at $c_{D} \approx-4$. The $\alpha$ binding increases in strength by $0.2 \mathrm{MeV}$ for each unit increase in $c_{D}$, and so we find reasonable 
agreement for all values of $c_{D} \sim O(1)$. If instead we fit $c_{E}$ according to the pseudo triton energy, then the lines in Fig. 9 shift downward in energy by about $2 \mathrm{MeV}$. The pseudo $\alpha$ energy with $p p$ - and $n n$-forces matched to $n p$-forces is estimated to be $-29.8(1) \mathrm{MeV}$ [33].

\section{SUMMARY AND DISCUSSION}

We have presented the first study of low-energy few-nucleon systems on the lattice at next-to-next-to-leading order in chiral effective field theory. We computed nucleon-nucleon phase shifts and $S$ - $D$ mixing angle on the lattice and used scattering data to determine unknown two-nucleon operator coefficients. In the three-nucleon system we calculated the triton energy and determined neutron-deuteron phase shifts using Lüscher's finite volume method. These were used to constrain the two cutoff-dependent three-body coefficients, $c_{D}$ and $c_{E}$. For the four-nucleon system we recast the lattice action in terms of auxiliary fields and used projection Monte Carlo to calculate the energy of the $\alpha$ particle.

There are several ways in which the lattice calculations presented here can be improved and extended in future work. One improvement is the inclusion of isospin-breaking effects due to Coulomb interactions and quark mass differences. The framework for isospinviolating effects in chiral effective field theory has been developed over the past decade [60, 61, 62, 63, 64, 65, 66, 67, 68]. Work is currently underway to implement these effects within the lattice formalism.

Another area of improvement concerns the $P$-wave phase shifts for our leading-order lattice action. NLO corrections to the phase shifts are substantial for nucleon momenta above $100 \mathrm{MeV}$. This seems not to cause any problems for the light $S$-shell nuclei considered here. However for $P$-shell nuclei we may find corrections strong enough to spoil the perturbative treatment of higher-order effects. In Ref. [7] this problem has already been resolved in neutron matter calculations using a new leading-order action $\mathrm{LO}_{3}$. This lattice action uses spin-isospin projection operators to produce Gaussian smearing only in even partial wave channels. The implementation of the $\mathrm{LO}_{3}$ action in Monte Carlo simulations with both protons and neutrons is computationally more intensive than the pure neutron simulations in Ref. [7]. The auxiliary-field formalism requires a total of 16 auxiliary fields and some increase in the sign/phase cancellations relative to the $\mathrm{LO}_{2}$ action. Nevertheless studies of light $P$-shell nuclei using the $\mathrm{LO}_{3}$ action are planned in the near future. 
One recent paper constrains the cutoff-dependent coefficient $c_{D}$ from the triton beta decay rate [69]. From the point of view of computing the spectrum of light nuclei, the easiest method for determining $c_{D}$ is by means of the $\alpha$-particle energy. This has the added benefit of removing systematic errors from the four nucleon system. If however we also want to accurately describe the chiral interactions of nucleons and light nuclei with soft pions, then it would be best to measure $c_{D}$ directly from the $\mathrm{SU}(2)$ axial coupling to two nucleon states. This is studied in Ref. [70] using pion production data in $p p$ scattering. Unfortunately the pion production energy threshold is too high to be accessible at our lattice spacing and extrapolations are required. In the future another approach may be possible using direct theory-to-theory matching. In this technique lattice QCD would be used to calculate the axial charge of two-neutron scattering states in a periodic cube. This calculated value of the axial charge could then be used to fix $c_{D}$ for any chosen lattice spacing in lattice chiral effective field theory.

\section{ACKNOWLEDGEMENTS}

We are grateful for discussions with Hans Hammer and Simon Kreuzer. Partial financial support from the Deutsche Forschungsgemeinschaft (SFB/TR 16), Helmholtz Association (contract number VH-NG-222 and VH-VI-231), and U.S. Department of Energy (DE-FG0203ER41260) are acknowledged. This work was further supported by the EU HadronPhysics2 project "Study of strongly interacting matter". The computational resources for this project were provided by the Jülich Supercomputing Centre at the Forschungszentrum Jülich.

\section{APPENDIX: LATTICE NOTATION}

The vector $\vec{n}$ represents integer-valued lattice vectors on a three-dimensional spatial lattice, and $\vec{p}, \vec{q}, \vec{k}$ represent integer-valued momentum lattice vectors. $\hat{l}=\hat{1}, \hat{2}, \hat{3}$ are unit lattice vectors in the spatial directions, $a$ is the spatial lattice spacing, and $L$ is the length of the cubic spatial lattice in each direction. The lattice time step is $a_{t}$, and $n_{t}$ labels the number of time steps. We define $\alpha_{t}$ as the ratio between lattice spacings, $\alpha_{t}=a_{t} / a$. Throughout our lattice discussion we use dimensionless parameters and operators, which correspond with physical values multiplied by the appropriate power of $a$. Final results are 
presented in physical units with the corresponding unit stated explicitly.

We use $a$ and $a^{\dagger}$ to denote annihilation and creation operators. We make explicit all spin and isospin indices,

$$
\begin{aligned}
& a_{0,0}=a_{\uparrow, p}, \quad a_{0,1}=a_{\uparrow, n}, \\
& a_{1,0}=a_{\downarrow, p}, \quad a_{1,1}=a_{\downarrow, n} .
\end{aligned}
$$

The first subscript is for spin and the second subscript is for isospin. We use $\tau_{I}$ with $I=1,2,3$ to represent Pauli matrices acting in isospin space and $\sigma_{S}$ with $S=1,2,3$ to represent Pauli matrices acting in spin space.

We use the eight vertices of a unit cube on the lattice to define spatial derivatives. For each spatial direction $l=1,2,3$ and any lattice function $f(\vec{n})$, let

$$
\Delta_{l} f(\vec{n})=\frac{1}{4} \sum_{\nu_{1}, \nu_{2}, \nu_{3}=0,1}(-1)^{\nu_{l}+1} f(\vec{n}+\vec{\nu}), \quad \vec{\nu}=\nu_{1} \hat{1}+\nu_{2} \hat{2}+\nu_{3} \hat{3} .
$$

We also define the double spatial derivative along direction $l$,

$$
\nabla_{l}^{2} f(\vec{n})=f(\vec{n}+\hat{l})+f(\vec{n}-\hat{l})-2 f(\vec{n})
$$

For the three-body NNLO interactions we also use the notation

$$
\square f(\vec{n})=\frac{1}{8} \sum_{\nu_{1}, \nu_{2}, \nu_{3}=0,1} f(\vec{n}+\vec{\nu}), \quad \vec{\nu}=\nu_{1} \hat{1}+\nu_{2} \hat{2}+\nu_{3} \hat{3} .
$$

\section{Local densities and currents}

We define the local density,

$$
\rho^{a^{\dagger}, a}(\vec{n})=\sum_{i, j=0,1} a_{i, j}^{\dagger}(\vec{n}) a_{i, j}(\vec{n})
$$

which is invariant under Wigner's SU(4) symmetry [39]. Similarly we define the local spin density for $S=1,2,3$,

$$
\rho_{S}^{a^{\dagger}, a}(\vec{n})=\sum_{i, j, i^{\prime}=0,1} a_{i, j}^{\dagger}(\vec{n})\left[\sigma_{S}\right]_{i i^{\prime}} a_{i^{\prime}, j}(\vec{n}),
$$

isospin density for $I=1,2,3$,

$$
\rho_{I}^{a^{\dagger}, a}(\vec{n})=\sum_{i, j, j^{\prime}=0,1} a_{i, j}^{\dagger}(\vec{n})\left[\tau_{I}\right]_{j j^{\prime}} a_{i, j^{\prime}}(\vec{n})
$$


and spin-isospin density for $S, I=1,2,3$,

$$
\rho_{S, I}^{a^{\dagger}, a}(\vec{n})=\sum_{i, j, i^{\prime}, j^{\prime}=0,1} a_{i, j}^{\dagger}(\vec{n})\left[\sigma_{S}\right]_{i i^{\prime}}\left[\tau_{I}\right]_{j j^{\prime}} a_{i^{\prime}, j^{\prime}}(\vec{n}) .
$$

For each static density we also have an associated current density. Similar to the definition of the lattice derivative $\Delta_{l}$ in Eq. (A.3), we use the eight vertices of a unit cube,

$$
\vec{\nu}=\nu_{1} \hat{1}+\nu_{2} \hat{2}+\nu_{3} \hat{3}
$$

for $\nu_{1}, \nu_{2}, \nu_{3}=0,1$. Let $\vec{\nu}(-l)$ for $l=1,2,3$ be the result of reflecting the $l^{\text {th }}$-component of $\vec{\nu}$ about the center of the cube,

$$
\vec{\nu}(-l)=\vec{\nu}+\left(1-2 \nu_{l}\right) \hat{l}
$$

Omitting factors of $i$ and $1 / m$, we can write the $l^{\text {th }}$-component of the SU(4)-invariant current density as

$$
\Pi_{l}^{a^{\dagger}, a}(\vec{n})=\frac{1}{4} \sum_{\nu_{1}, \nu_{2}, \nu_{3}=0,1} \sum_{i, j=0,1}(-1)^{\nu_{l}+1} a_{i, j}^{\dagger}(\vec{n}+\vec{\nu}(-l)) a_{i, j}(\vec{n}+\vec{\nu}) .
$$

Similarly the $l^{\text {th }}$-component of spin current density is

$$
\Pi_{l, S}^{a^{\dagger}, a}(\vec{n})=\frac{1}{4} \sum_{\nu_{1}, \nu_{2}, \nu_{3}=0,1} \sum_{i, j, i^{\prime}=0,1}(-1)^{\nu_{l}+1} a_{i, j}^{\dagger}(\vec{n}+\vec{\nu}(-l))\left[\sigma_{S}\right]_{i i^{\prime}} a_{i^{\prime}, j}(\vec{n}+\vec{\nu}),
$$

$l^{\text {th }}$-component of isospin current density is

$$
\Pi_{l, I}^{a^{\dagger}, a}(\vec{n})=\frac{1}{4} \sum_{\nu_{1}, \nu_{2}, \nu_{3}=0,1} \sum_{i, j, j^{\prime}=0,1}(-1)^{\nu_{l}+1} a_{i, j}^{\dagger}(\vec{n}+\vec{\nu}(-l))\left[\tau_{I}\right]_{j j^{\prime}} a_{i, j^{\prime}}(\vec{n}+\vec{\nu}),
$$

and $l^{\text {th }}$-component of spin-isospin current density is

$$
\Pi_{l, S, I}^{a^{\dagger}, a}(\vec{n})=\frac{1}{4} \sum_{\nu_{1}, \nu_{2}, \nu_{3}=0,1} \sum_{i, j, i^{\prime}, j^{\prime}=0,1}(-1)^{\nu_{l}+1} a_{i, j}^{\dagger}(\vec{n}+\vec{\nu}(-l))\left[\sigma_{S}\right]_{i i^{\prime}}\left[\tau_{I}\right]_{j j^{\prime}} a_{i^{\prime}, j^{\prime}}(\vec{n}+\vec{\nu}) .
$$

\section{Instantaneous free pion action}

The lattice action for free pions with purely instantaneous propagation is

$$
S_{\pi \pi}\left(\pi_{I}\right)=\alpha_{t}\left(\frac{m_{\pi}^{2}}{2}+3\right) \sum_{\vec{n}, n_{t}, I} \pi_{I}\left(\vec{n}, n_{t}\right) \pi_{I}\left(\vec{n}, n_{t}\right)-\alpha_{t} \sum_{\vec{n}, n_{t}, I, l} \pi_{I}\left(\vec{n}, n_{t}\right) \pi_{I}\left(\vec{n}+\hat{l}, n_{t}\right),
$$

where $\pi_{I}$ is the pion field labelled with isospin index $I$. It is convenient to define a rescaled pion field, $\pi_{I}^{\prime}$,

$$
\pi_{I}^{\prime}\left(\vec{n}, n_{t}\right)=\sqrt{q_{\pi}} \pi_{I}\left(\vec{n}, n_{t}\right)
$$




$$
q_{\pi}=\alpha_{t}\left(m_{\pi}^{2}+6\right)
$$

Then

$$
S_{\pi \pi}\left(\pi_{I}^{\prime}\right)=\frac{1}{2} \sum_{\vec{n}, n_{t}, I} \pi_{I}^{\prime}\left(\vec{n}, n_{t}\right) \pi_{I}^{\prime}\left(\vec{n}, n_{t}\right)-\frac{\alpha_{t}}{q_{\pi}} \sum_{\vec{n}, n_{t}, I, l} \pi_{I}^{\prime}\left(\vec{n}, n_{t}\right) \pi_{I}^{\prime}\left(\vec{n}+\hat{l}, n_{t}\right)
$$

In momentum space the action is

$$
S_{\pi \pi}\left(\pi_{I}^{\prime}\right)=\frac{1}{L^{3}} \sum_{I, \vec{k}} \pi_{I}^{\prime}\left(-\vec{k}, n_{t}\right) \pi_{I}^{\prime}\left(\vec{k}, n_{t}\right)\left[\frac{1}{2}-\frac{\alpha_{t}}{q_{\pi}} \sum_{l} \cos k_{l}\right] .
$$

The instantaneous pion correlation function at spatial separation $\vec{n}$ is

$$
\begin{aligned}
\left\langle\pi_{I}^{\prime}\left(\vec{n}, n_{t}\right) \pi_{I}^{\prime}\left(\overrightarrow{0}, n_{t}\right)\right\rangle & \left.=\frac{\int D \pi_{I}^{\prime} \pi_{I}^{\prime}\left(\vec{n}, n_{t}\right) \pi_{I}^{\prime}\left(\overrightarrow{0}, n_{t}\right) \exp \left[-S_{\pi \pi}\right]}{\int D \pi_{I}^{\prime} \exp \left[-S_{\pi \pi}\right]} \text { (no sum on } I\right) \\
& =\frac{1}{L^{3}} \sum_{\vec{k}} e^{-i \vec{k} \cdot \vec{n}} D_{\pi}(\vec{k})
\end{aligned}
$$

where

$$
D_{\pi}(\vec{k})=\frac{1}{1-\frac{2 \alpha_{t}}{q_{\pi}} \sum_{l} \cos k_{l}} .
$$

It is also useful to define the two-derivative pion correlator, $G_{S_{1} S_{2}}(\vec{n})$,

$$
\begin{aligned}
G_{S_{1} S_{2}}(\vec{n}) & =\left\langle\Delta_{S_{1}} \pi_{I}^{\prime}\left(\vec{n}, n_{t}\right) \Delta_{S_{2}} \pi_{I}^{\prime}\left(\overrightarrow{0}, n_{t}\right)\right\rangle \quad(\text { no sum on } I) \\
& =\frac{1}{16} \sum_{\nu_{1}, \nu_{2}, \nu_{3}=0,1} \sum_{\nu_{1}^{\prime}, \nu_{2}^{\prime}, \nu_{3}^{\prime}=0,1}(-1)^{\nu_{S_{1}}}(-1)^{\nu_{S_{2}}^{\prime}}\left\langle\pi_{I}^{\prime}\left(\vec{n}+\vec{\nu}-\vec{\nu}^{\prime}, n_{t}\right) \pi_{I}^{\prime}\left(\overrightarrow{0}, n_{t}\right)\right\rangle
\end{aligned}
$$

[1] S. Weinberg, Phys. Lett. B251, 288 (1990).

[2] S. Weinberg, Nucl. Phys. B363, 3 (1991).

[3] D. Lee, B. Borasoy, and T. Schäfer, Phys. Rev. C70, 014007 (2004), nucl-th/0402072.

[4] B. Borasoy, E. Epelbaum, H. Krebs, D. Lee, and U.-G. Meißner, Eur. Phys. J. A31, 105 (2007), nucl-th/0611087.

[5] B. Borasoy, E. Epelbaum, H. Krebs, D. Lee, and U.-G. Meißner, Eur. Phys. J. A35, 343 (2008), arXiv:0712.2990 [nucl-th].

[6] B. Borasoy, E. Epelbaum, H. Krebs, D. Lee, and U.-G. Meißner, Eur. Phys. J. A35, 357 (2008), arXiv:0712.2993 [nucl-th].

[7] E. Epelbaum, H. Krebs, D. Lee, and U.-G. Meißner (2008), 0812.3653. 
[8] U. van Kolck, Prog. Part. Nucl. Phys. 43, 337 (1999), nucl-th/9902015.

[9] P. F. Bedaque and U. van Kolck, Ann. Rev. Nucl. Part. Sci. 52, 339 (2002), nucl-th/0203055.

[10] E. Epelbaum, Prog. Part. Nucl. Phys. 57, 654 (2006), nucl-th/0509032.

[11] E. Epelbaum, H.-W. Hammer, and U.-G. Meißner (2008), arXiv:0811.1338 [nucl-th].

[12] B. Borasoy, E. Epelbaum, H. Krebs, D. Lee, and U.-G. Meißner, Eur. Phys. J. A34, 185 (2007), arXiv:0708.1780 [nucl-th].

[13] C. Ordonez and U. van Kolck, Phys. Lett. B291, 459 (1992).

[14] C. Ordonez, L. Ray, and U. van Kolck, Phys. Rev. Lett. 72, 1982 (1994).

[15] C. Ordonez, L. Ray, and U. van Kolck, Phys. Rev. C53, 2086 (1996), hep-ph/9511380.

[16] E. Epelbaum, W. Glockle, and U.-G. Meißner, Nucl. Phys. A637, 107 (1998), nucl-th/9801064.

[17] E. Epelbaum, W. Gloeckle, and U.-G. Meißner, Nucl. Phys. A671, 295 (2000), nuclth/9910064.

[18] J. L. Friar and S. A. Coon, Phys. Rev. C49, 1272 (1994).

[19] N. Kaiser, R. Brockmann, and W. Weise, Nucl. Phys. A625, 758 (1997), nucl-th/9706045.

[20] V. Bernard, N. Kaiser, and U.-G. Meißner, Int. J. Mod. Phys. E4, 193 (1995), hep-ph/9501384.

[21] P. Büttiker and U.-G. Meißner, Nucl. Phys. A668, 97 (2000), hep-ph/9908247.

[22] J. Fujita and H. Miyazawa, Prog. Theor. Phys. 17, 360 (1957).

[23] B. H. J. McKellar and R. Rajaraman, Phys. Rev. Lett. 21, 450 (1968).

[24] S.-N. Yang, Phys. Rev. C10, 2067 (1974).

[25] S. A. Coon, M. D. Scadron, and B. R. Barrett, Nucl. Phys. A242, 467 (1975).

[26] S. A. Coon et al., Nucl. Phys. A317, 242 (1979).

[27] S. A. Coon and W. Gloeckle, Phys. Rev. C23, 1790 (1981).

[28] J. Carlson, V. R. Pandharipande, and R. B. Wiringa, Nucl. Phys. A401, 59 (1983).

[29] H. T. Coelho, T. K. Das, and M. R. Robilotta, Phys. Rev. C28, 1812 (1983).

[30] B. S. Pudliner, V. R. Pandharipande, J. Carlson, S. C. Pieper, and R. B. Wiringa, Phys. Rev. C56, 1720 (1997), nucl-th/9705009.

[31] U. van Kolck, Phys. Rev. C49, 2932 (1994).

[32] J. L. Friar, D. Huber, and U. van Kolck, Phys. Rev. C59, 53 (1999), nucl-th/9809065.

[33] E. Epelbaum, A. Nogga, W. Glöckle, H. Kamada, U.-G. Meißner, and H. Witala, Phys. Rev. C66, 064001 (2002), nucl-th/0208023.

[34] S. R. Beane, P. F. Bedaque, M. J. Savage, and U. van Kolck, Nucl. Phys. A700, 377 (2002), 
nucl-th/0104030.

[35] A. Nogga, R. G. E. Timmermans, and U. van Kolck, Phys. Rev. C72, 054006 (2005), nuclth/0506005.

[36] M. C. Birse, Phys. Rev. C74, 014003 (2006), nucl-th/0507077.

[37] M. C. Birse, Phys. Rev. C76, 034002 (2007), arXiv:0706.0984 [nucl-th].

[38] E. Epelbaum and U.-G. Meißner (2006), nucl-th/0609037.

[39] E. Wigner, Phys. Rev. 51, 106 (1937).

[40] P. F. Bedaque, H.-W. Hammer, and U. van Kolck, Phys. Rev. Lett. 82, 463 (1999), nuclth/9809025.

[41] P. F. Bedaque, H.-W. Hammer, and U. van Kolck, Nucl. Phys. A646, 444 (1999), nuclth/9811046.

[42] P. F. Bedaque, H.-W. Hammer, and U. van Kolck, Nucl. Phys. A676, 357 (2000), nuclth/9906032.

[43] L. H. Thomas, Phys. Rev. 47, 903 (1935).

[44] J.-W. Chen, D. Lee, and T. Schäfer, Phys. Rev. Lett. 93, 242302 (2004), nucl-th/0408043.

[45] B. Borasoy, H. Krebs, D. Lee, and U.-G. Meißner, Nucl. Phys. A768, 179 (2006), nuclth/0510047.

[46] D. Lee (2008), arXiv:0804.3501 [nucl-th].

[47] D. Lee, Phys. Rev. A73, 063204 (2006), physics/0512085.

[48] M. Lüscher, Commun. Math. Phys. 104, 177 (1986).

[49] P. Navratil et al., Few Body Syst. 43, 129 (2008), 0712.1207.

[50] M. Lüscher, Commun. Math. Phys. 105, 153 (1986).

[51] M. Lüscher, Nucl. Phys. B354, 531 (1991).

[52] K. Schoen et al. (2003), nucl-ex/0306012.

[53] H. Witala et al. (2003), nucl-th/0305028.

[54] W. T. H. van Oers and J. D. Seagrave, Phys. Lett. B24, 562 (1967).

[55] V. N. Efimov, Sov. J. Nucl. Phys. 12, 589 (1971).

[56] V. N. Efimov, Phys. Rev. C47, 1876 (1993).

[57] S. Kreuzer and H. W. Hammer (2008), 0811.0159.

[58] D. Lee, Phys. Rev. B73, 115112 (2006), cond-mat/0511332.

[59] D. Lee, Phys. Rev. B75, 134502 (2007), cond-mat/0606706. 
[60] U. van Kolck, J. L. Friar, and J. T. Goldman, Phys. Lett. B371, 169 (1996), nucl-th/9601009.

[61] U. van Kolck, M. C. M. Rentmeester, J. L. Friar, J. T. Goldman, and J. J. de Swart, Phys. Rev. Lett. 80, 4386 (1998), nucl-th/9710067.

[62] J. L. Friar and U. van Kolck, Phys. Rev. C60, 034006 (1999), nucl-th/9906048.

[63] E. Epelbaum and U.-G. Meißner, Phys. Lett. B461, 287 (1999), nucl-th/9902042.

[64] M. Walzl, U. G. Meißner, and E. Epelbaum, Nucl. Phys. A693, 663 (2001), nucl-th/0010019.

[65] J. L. Friar, U. van Kolck, G. L. Payne, and S. A. Coon, Phys. Rev. C68, 024003 (2003), nucl-th/0303058.

[66] J. L. Friar, U. van Kolck, M. C. M. Rentmeester, and R. G. E. Timmermans, Phys. Rev. C70, 044001 (2004), nucl-th/0406026.

[67] E. Epelbaum, U.-G. Meißner, and J. E. Palomar, Phys. Rev. C71, 024001 (2005), nuclth/0407037.

[68] E. Epelbaum and U.-G. Meißner, Phys. Rev. C72, 044001 (2005), nucl-th/0502052.

[69] D. Gazit, S. Quaglioni, and P. Navratil (2008), 0812.4444.

[70] C. Hanhart, U. van Kolck, and G. A. Miller, Phys. Rev. Lett. 85, 2905 (2000), nucl-th/0004033. 